\title{
Infinite-Dimensional Linear Algebra and Solvability of Partial Differential Equations
}

\author{
TODOR D. TODOROV
}

\begin{abstract}
We discuss linear algebra of infinite-dimensional vector spaces in terms of algebraic (Hamel) bases. As an application we prove the surjectivity of a large class of linear partial differential operators with smooth $\left(\mathcal{C}^{\infty}\right.$-coefficients) coefficients, called in the article regular, acting on the algebraic dual $\mathcal{D}^{*}(\Omega)$ of the space of test-functions $\mathcal{D}(\Omega)$. The surjectivity of the partial differential operators guarantees solvability of the corresponding partial differential equations within $\mathcal{D}^{*}(\Omega)$. We discuss our result in contrast to and comparison with similar results about the restrictions of the regular operators on the space of Schwartz distribution $\mathcal{D}^{\prime}(\Omega)$, where these operators are often non-surjective.
\end{abstract}

2020 Mathematics Subject Classification 15A03, 35A01, 35D35, 35E20, 35J15, 46F05, 46F10, 47F05 (primary); 46S20, 46F30 (secondary)

Keywords: Algebraic basis, Hamel basis, algebraic dual, dual operator, linear partial differential operator, linear partial differential equation, solvability, existence of a solution, generalized solution, Schwartz distribution

\section{Introduction}

In Sections 3-5 we present the basic results of infinite-dimensional linear algebra, an old branch of mathematics initiated in 1905 by Georg Hamel [12], dealing with infinitedimensional vector spaces in terms of algebraic (Hamel) bases rather than topological or orthonormal Hilbert bases. The approach is mostly algebraic. In Theorem 5.4 we show that a linear operator is injective if and only if its dual operator is surjective; a result well-known for finite-dimensional vector spaces but less-known for infinite-dimensional spaces. This gives rise to the Definition 5.5 of a regular linear operator-a surjective operator on the dual space with injective co-dual.

Several discussions of the earlier versions of this text convinced us that the algebraic (Hamel) bases have gradually been falling out of popularity in the last several decades. That is why the first part of the article (Sections 3-5) is written in somewhat tutorial manner, with many illustrative examples (Section 7). A reader who knows Theorem 5.4 
from the finite-dimensional linear algebra and who believes in its validity for infinitedimensional vector spaces might skip reading the first several sections and start directly from Section 8.

In Sections 8 we apply infinite-dimensional linear algebra to the particular case of the vector space $\mathcal{D}(\Omega)$ and its algebraic dual $\mathcal{D}^{*}(\Omega)$. Here $\Omega$ is an open set of $\mathbb{R}^{d}$ in the usual topology of $\mathbb{R}^{d}$. Somewhere in this section we abandon the realm of algebra and start involving concepts and methods from functional analysis and the theory of partial differential operators (Hörmander [15]-[17]). In particular, Definition 5.5 (mentioned above) — if applied to $\mathcal{D}(\Omega)$ - gives rise to the concept of regular operator with $\mathcal{C}^{\infty}$-coefficients: a surjective linear operator $P^{*}(x, \partial)$ with $\mathcal{C}^{\infty}$-coefficients acting on $\mathcal{D}^{*}(\Omega)$ which has an injective co-dual (transposed) operator $P(x, \partial)$ on $\mathcal{D}(\Omega)$.

For readers without background in the Schwartz theory of distributions (Vladimirov [39]) who are otherwise interested in the main topic of our article, we offer a characterization of the space of Schwartz distributions $\mathcal{D}^{\prime}(\Omega)$ as a particular subspace of $\mathcal{D}^{*}(\Omega)$ without the usual involvement of the strong topology on the space of test-functions $\mathcal{D}(\Omega)$ (Section 9). We shortly outline a sequential approach to distribution theory based on our characterization (Remark 9.2). Thus, the dilemma $\mathcal{D}^{\prime}(\Omega)$ vs. $\mathcal{D}^{*}(\Omega)$-discussed in Section 11- can be followed by readers without strong (or any) background in the Schwartz theory of distributions.

In Section 11 we identify several subclasses of linear partial differential operators in mathematics (Hörmander [15]-[17]) as regular (thus, surjective on $\mathcal{D}^{*}(\Omega)$ ) which include the following:

- All linear partial differential operators with constant coefficients are regular.

- The Hans Lewy [23] operator: $L^{*}(x, \partial)=\frac{\partial}{\partial x_{1}}+i \frac{\partial}{\partial x_{2}}-2 i\left(x_{1}+i x_{2}\right) \frac{\partial}{\partial x_{3}}$ is regular.

- All second order elliptic operators with $\mathcal{C}^{\infty}$-coefficients are regular.

- All elliptic operators with analytic coefficients are regular.

All of these operators are surjective on $\mathcal{D}^{*}(\Omega)$, but not necessarily surjective on the following three invariant subspaces $\mathcal{D}(\Omega), \mathcal{E}(\Omega)$ and $\mathcal{D}^{\prime}(\Omega)$ (Section 10). Consequently, we prove the solvability of the partial differential equations of the form $P^{*}(x, \partial) U=T$ in $\mathcal{D}^{*}(\Omega)$, for regular operators $P^{*}(x, \partial): \mathcal{D}^{*}(\Omega) \mapsto \mathcal{D}^{*}(\Omega)$. In other words, we prove the existence of a solution $U$ in $\mathcal{D}^{*}(\Omega)$ for every choice of $T$ also in $\mathcal{D}^{*}(\Omega)$. We should recall that:

- Every linear partial differential operator with constant coefficients $P^{*}(\partial)$ on $\mathcal{D}^{\prime}\left(\mathbb{R}^{d}\right)$ is surjective; this is the famous existence theorem of Malgrange [28] and Ehrenpreis [9].

- The Malgrange-Ehrenpreis existence result might, however, fail in $\mathcal{D}^{\prime}(\Omega)$ for operators which are hypoelliptic but not elliptic, subsets $\Omega$ of $\mathbb{R}^{d}$ which are 
open, but not P-convex for supports (Hörmander [14, Theorem 10.6.6, Corollary 10.6.8]). Thus, the partial differential equation $P^{*}(\partial) U=f$ might have no solutions in $\mathcal{D}^{\prime}(\Omega)$ even for some smooth $f$.

- Hans Lewy [23] was the first to show that the Lewy operator $L^{*}(x, \partial)$ is not surjective on $\mathcal{D}^{\prime}\left(\mathbb{R}^{3}\right)$. Thus, the partial differential equations of the form $L^{*}(x, \partial) U=\varphi$ might fail to have solution $U$ in $\mathcal{D}^{\prime}\left(\mathbb{R}^{3}\right)$ even for some testfunctions $\varphi \in \mathcal{D}\left(\mathbb{R}^{3}\right)$. A general existence result also fails in the space of hyperfunctions (Schapira [35]).

- The elliptic operators mentioned above are, in general, also non-surjective on $\mathcal{D}^{\prime}(\Omega)$.

In Section 12 we show-with the help of Hamel bases-that the space of generalized distributions $\widehat{\mathcal{E}}(\Omega)$ introduced in Todorov $[37, \S 2]$ can be embedded as a $\mathbb{C}$-vector subspace into the algebraic dual $\mathcal{D}^{*}(\Omega)$ of the space of test-functions $\mathcal{D}(\Omega)$. Because $\widehat{\mathcal{E}}(\Omega)$ was defined in the framework of nonstandard analysis (Robinson [33]), we look upon $\mathcal{D}^{*}(\Omega)$ as a standardization of $\widehat{\mathcal{E}}(\Omega)$. Actually, our article itself can be viewed as a standardization of the results in [37], because the surjectivity of the regular operators was first proved in [37] in the framework of $\mathcal{L}(\widehat{\mathcal{E}}(\Omega))$, while the main result of this article (Theorem 8.4) holds within $\mathcal{L}\left(\mathcal{D}^{*}(\Omega)\right.$ ). Thus, by replacing $\widehat{\mathcal{E}}(\Omega)$ with $\mathcal{D}^{*}(\Omega)$, our result about the regular operators becomes accessible even for readers without background in nonstandard analysis. Our standardization is, of course, not an isolated event in mathematics; we remind two more cases of standardizations in the history of mathematics.

Our inspiration comes from the Fundamental Theorem of Algebra: following this analogy the space $\mathcal{D}^{\prime}(\Omega)$ is the counterpart of the field of real numbers $\mathbb{R}$, the space $\mathcal{D}^{*}(\Omega)$ is the counterpart of the field of complex numbers $\mathbb{C}$, and the class of regular operator is the counterpart of the ring of polynomials $\mathbb{C}[x]$. We are trying to convince the reader that the space $\mathcal{D}^{*}(\Omega)$-rather than $\mathcal{D}^{\prime}(\Omega)$ —deserves to be considered as the natural theoretical framework for the class of regular operators $P^{*}(x, \partial)$, since the equations of the form $P^{*}(x, \partial) U=T$ often have no solutions in $\mathcal{D}^{\prime}(\Omega)$.

Recall as well that the global solvability of arbitrary analytic partial differential equations was studied in Rosinger [34, Chapter 2] and Oberguggenberger [29, Section 22]. The existence results for continuous partial differential operators are obtained by means of the Dedekind order completion method in Oberguggenberger and Rosinger [31].

A general solvability of evolution-type equations appears in Colombeau, Heibig and Oberguggenberger [7], using regularized derivatives in the framework of Colombeau algebra of generalized functions (see Colombeau [6] and Oberguggenberger [29]). 
As we mentioned above, the surjectivity of the regular operators was first proved in Todorov [37] in the framework of nonstandard analysis. Meanwhile (in the period between the publication of [37] and the writing of this article) two more similar articles in the framework of $\mathcal{D}^{*}(\Omega)$ appeared: an unpublished manuscript Oberguggenberger and Todorov [32] and Oberguggenberger [30]. In this article we shall use some of the results in [30].

Finally, we should mention that our article has somewhat an ideological edge because we challenge at least two widely spread prejudices in the mathematical community. The first one is that Hamel bases are not and can never be mathematically useful. The second one is that we should never go beyond the space of Schwartz distributions $\mathcal{D}^{\prime}(\Omega)$ as a framework of a partial differential equation, especially if the equation is linear. That is to say, "better to admit (perhaps with some regret) that a given equation has no solutions rather than look for a solution outside $\mathcal{D}^{\prime}(\Omega)$ ".

This article is dedicated to Professor Michael Oberguggenberger on occasion of his 65 th birthday.

\section{Notations and set-theoretical framework}

The set-theoretical framework of this text is the usual ZFC-axioms (Zermelo-Fraenkel axioms with the Axiom of Choice) along with the GCH (Generalized Continuum Hypothesis) in the form $2^{\kappa}=\kappa_{+}$for every cardinal $\kappa$ (or equivalently, $2^{\aleph \alpha}=\aleph_{\alpha+1}$ for all ordinals $\alpha$ ). Here we write $\kappa_{+}$for the successor of $\kappa$. For the domain of ZFC and GCH axioms we use the superstructures $\widehat{\mathcal{S}}$ with the set of individuals $\mathcal{S}=\mathbb{K} \cup V$, where $V$ is the vector space over a field $\mathbb{K}$ under consideration (eg $V=\mathbb{R}^{n}$ with $\mathbb{K}=\mathbb{R}$ or $V=\mathbb{C}^{n}$ with $\mathbb{K}=\mathbb{C}$, etc). Our formal language is based on bounded quantifiers of the form $(\forall x \in A) \alpha(x)$ and $(\exists x \in B) \beta(x)$, where $A, B \in \widehat{\mathcal{S}} \backslash \mathcal{S}$ and $\alpha(x)$ and $\beta(x)$ are predicates (Davis [8, pages 11-15]). We believe however, that the rest of this text can be followed without a familiarity with the concept of superstructure.

We recall that the following are equivalent (Wolf [40, page 255] and/or Jech [20, Chapter 11, A1]):

- Axiom of Choice.

- Zorn's Lemma.

- Every set can be well-ordered.

- The usual partial order on the class of cardinal numbers is a total order. 
In particular, Zorn's Lemma will be involved in Theorem 3.6 and the total order between cardinals is needed in the proof of Lemma 3.9. Also, "every set can be well-ordered" will be useful to supply a basis with a well-ordering if desired (Remark 6.4).

Actually, we do not need the GCH except for the purpose of simplifying the calculations with cardinals and the dimension of vector spaces. For example, with the help of GCH, $\aleph_{0}<\operatorname{dim}(V) \leq \mathfrak{c}$ implies simply $\operatorname{dim}(V)=\mathfrak{c}$ (rather than only $\left.\operatorname{dim}(V)=\aleph_{1}\right)$. Here $\aleph_{0}=\operatorname{card}(\mathbb{N})$ and $\mathfrak{c}=\operatorname{card}(\mathbb{R})$.

If $X$ is a set, we shall treat $X$ as a subset of the power set $\mathcal{P}(X)$, in symbols, $X \subset \mathcal{P}(X)$ by means of the embedding $x \mapsto\{x\}$. If $X$ and $Y$ are two sets, we denote by $Y^{X}$ the set of all functions from $X$ to $Y$.

For index sets (for indexing bases, for example) we use the popular sets: $\mathbb{N}, \mathbb{R}, \mathbb{R}^{d}$, $\mathcal{P}(\mathbb{R}), \mathcal{P}\left(\mathbb{R}^{d}\right), \mathcal{P}(\mathcal{P}(\mathbb{R}))$, etc with cardinalities $\aleph_{0}, \mathfrak{c}, \mathfrak{c}, \mathfrak{c}_{+}, \mathfrak{c}_{+},\left(\mathfrak{c}_{+}\right)_{+}$, respectively. We sometimes use the field of scalars $\mathbb{K}$ itself as an index set or $\mathbb{K}^{d}, \mathcal{P}(\mathbb{K}), \mathcal{P}\left(\mathbb{K}^{d}\right), \mathcal{P}(\mathcal{P}(\mathbb{K}))$, etc.

In what follows $V$ stands for a generic vector space over a field $\mathbb{K}$ (Axler [2]). Sometimes we shall write $V \mid \mathbb{K}$ instead of $V$. If we write $U \subseteq V$, we mean that both $U$ and $V$ are vector spaces over the same field and $U$ is a vector subspace of $V$. Similarly, $V \cong W$ means that $V$ and $W$ are isomorphic vector spaces. $\mathcal{L}(V)$ denotes the $\mathbb{K}$-vector space consisting of all linear operators $L: V \mapsto V$. We denote by $V^{*}$ the algebraic dual of $V$. We denote by $T(v)$ or $\langle T, v\rangle$ the evaluation of $T \in V^{*}$ at $v \in V$.

Let $\mathcal{T}^{d}$ denote the usual topology on $\mathbb{R}^{d}$ and let $X, Y \in \mathcal{T}^{d}$ be two open set of $\mathbb{R}^{d}$. We denote by $\operatorname{Diff}(X, Y)$ the set of all diffeomorphisms from $X$ to $Y$. If $\theta \in \operatorname{Diff}(X, Y)$, we denote by $J_{\theta}: X \rightarrow \mathbb{R}, J_{\theta}=\left|\operatorname{det}\left(\frac{\partial \theta}{\partial x}\right)\right|$, the corresponding Jacobian determinant. We denote by $\operatorname{Diff}(X)$ the group of diffeomorphisms from $X$ to itself.

Let $\Omega$ stand for a (generic) open subset of $\mathbb{R}^{d}$. Here is a list of popular functional spaces and notations:

- $\mathcal{E}(\Omega)=\mathcal{C}^{\infty}(\Omega)$ denotes the space $\mathcal{C}^{\infty}$-functions from $\Omega$ to $\mathbb{C}$.

- $\mathcal{D}(\Omega)=\mathcal{C}_{0}^{\infty}(\Omega)$ denote the space of test-functions on $\Omega$, ie the $\mathcal{C}^{\infty}$-functions from $\Omega$ to $\mathbb{C}$ with compact support (Vladimirov [39]).

- $\mathcal{L}^{2}(\Omega)$ denotes the usual Hilbert space of Lebesgue measurable square integrable functions from $\Omega$ to $\mathbb{C}$.

- $\mathcal{L}^{\infty}(\Omega)$ denotes the space of Lebesgue measurable bounded functions from $\Omega$ to $\mathbb{C}$.

- $\mathcal{L}_{\text {loc }}(\Omega)$ stands for the Lebesgue measurable locally integrable functions from $\Omega$ to $\mathbb{C}$. 
- $\mathcal{D}^{\prime}(\Omega)$ denotes the space of Schwartz distributions on $\Omega$ (Vladimirov [39]).

- $\mathcal{E}^{\prime}(\Omega)$ denotes the space of Schwartz distributions with compact support.

- We denote by $\mathcal{D}^{*}(\Omega)$ and $\mathcal{E}^{*}(\Omega)$ the algebraic duals of $\mathcal{D}(\Omega)$ and $\mathcal{E}(\Omega)$, respectively.

\section{Infinite-dimensional linear algebra}

We recall the definitions of algebraic (Hamel) basis and dimension of an infinitedimensional vector space (Hamel [12]). For more details and the missing proofs we refer to Jacobson [19], Hungerford [18], Hewitt and Stromberg [13] and Mackey [27].

Definition 3.1 (Basis and Dimension) Let $V$ be a non-trivial vector space over a field $\mathbb{K}$.

(1) A subset $\mathcal{B}$ of $V$ is called free if every finite subset of $\mathcal{B}$ consists of linearly independent vectors in $V$.

(2) A free set $\mathcal{B}$ of $V$ is called maximal (or, a maximal free set) if $\mathcal{B}$ cannot be extended (properly) to a free set of $V$. Every maximal free set $\mathcal{B}$ of $V$ is called an algebraic basis, Hamel basis, or simply basis of $V$.

(3) If $\mathcal{B}$ is a basis of $V$, the dimension (or Hamel dimension) of $V$ is defined by $\operatorname{dim} V=\operatorname{card} \mathcal{B}$.

The definition of dimension is justified by the following result.

Lemma 3.2 (Justification) All bases of $V$ have the same cardinality.

Proof We refer to Jacobson [19, Chapter 9, §2, page 240], Hungerford [18, Theorem 2.6, page 184] or Hewitt and Stromberg [13, Theorem 4.58, page 30].

Lemma 3.3 (Span) Let $\mathcal{B}$ be a basis of $V$. Then every non-zero vector $v \in V$ can be uniquely presented as a (finite) linear combination of vectors in $\mathcal{B}$ with non-zero coefficients in $\mathbb{K}$. We summarize this in $V=\operatorname{span} \mathcal{B}$. Consequently, card $V=\max \{\operatorname{dim} V, \operatorname{card} \mathbb{K}\}$.

Proof Let $v \in V, v \neq 0$, and suppose (seeking a contradiction) that $v \neq \sum_{k=1}^{m} c_{n} v_{n}$ for all $m \in \mathbb{N}$, all $v_{n} \in \mathcal{B}$ and all $c_{n} \in \mathbb{K}$. Thus the set $\overline{\mathcal{B}}=\mathcal{B} \cup\{v\}$ is also a free set, contradicting the maximality of $\mathcal{B}$. 
Definition 3.4 (Spectrum) Let $\mathcal{B}=\left\{v_{s}: s \in S\right\}$ be a Hamel basis of $V$ with an index set $S$ with card $S=\operatorname{dim} V$. Let $v \in V, v \neq 0$. Then the (finite) set $\operatorname{sp}(v) \subset S$ is called the spectrum of $v$ (relative to $\mathcal{B}$ and $S$ ) if $v=\sum_{s \in \operatorname{sp}(v)} c_{s} v_{s}$ and $c_{s} \in \mathbb{K} \backslash\{0\}$ for all $s \in \operatorname{sp}(v)$. We shall sometimes write simply $v=\sum_{s \in S} c_{s} v_{s}$ or even $v=\sum c_{s} v_{s}$ instead of $v=\sum_{s \in \operatorname{sp}(v)} c_{s} v_{s}$ (keeping in mind that the sum $\sum_{s \in S} c_{s} v_{s}=\sum c_{s} v_{s}$ is finite). We also let $\operatorname{sp}(0)=\varnothing$ and $\sum_{s \in \varnothing} c_{s} v_{s}=0$.

Notice that $\operatorname{card} \mathbb{K} \leq \operatorname{card} V$ and $\operatorname{dim} V \leq \operatorname{card} V$ hold trivially. The next equalities follow immediately from the formula in Lemma 3.3.

Corollary 3.5 (Two Equalities) (1) If $\operatorname{card} \mathbb{K}<\operatorname{card} V$, then $\operatorname{dim} V=\operatorname{card} V$ (see Example 7.1 in this paper).

(2) If $\operatorname{dim} V<\operatorname{card} V$, then $\operatorname{card} V=\operatorname{card} \mathbb{K}$ (see Example 7.5 and Example 7.12).

Theorem 3.6 (Existence of Basis) Let $V$ be a vector space over a field of scalars $\mathbb{K}$ and let $E \subset V$ be a free set of $V$. Then there exists a basis $\mathcal{B}$ of $V$ which contains $E$ and such that $V=\operatorname{span} E \oplus \operatorname{span}(\mathcal{B} \backslash E)$. Consequently, every non-trivial vector space has a basis.

Proof Consider the family of subsets of $V$ :

$$
\mathcal{F}(E)=\{S \in \mathcal{P}(V): S \text { is a free set and } E \subseteq S\}
$$

We shall treat $\mathcal{F}(E)$ as a partially ordered set under the inclusion, $\subseteq$. Note that $\mathcal{F}(E)$ is a non-empty set, because $E \in \mathcal{F}(E)$. We observe that every totally ordered subset (chain) $\mathcal{C}$ of $\mathcal{F}(E)$ is bounded from above by its union $\bigcup_{C \in \mathcal{C}} C$ and also $\bigcup_{C \in \mathcal{C}} C \in \mathcal{F}(E)$. By Zorn's Lemma $\mathcal{F}(E)$ has a maximal element, $\mathcal{B}$.

Remark 3.7 (Hamel Bases) (1) We sometimes refer to the maximal free sets $\mathcal{B}$ of $V$ as Hamel bases of $V$ after Georg Hamel [12] who proved the above theorem in 1905 in the particular case of $V=\mathbb{R}$ and $\mathbb{K}=\mathbb{Q}$ (see Example 7.1 later in the paper).

(2) Unlike the case of finite-dimensional vector spaces, in the case of an infinitedimensional vector space $V$ the equality $\operatorname{card} E=\operatorname{dim} V$ for some free set $E$ of $V$ does not imply that $E$ is a basis of $V$. Indeed, let $\mathcal{B}$ be a basis of $V$ and let $E=\mathcal{B} \backslash\{v\}$ for some $v \in \mathcal{B}$. Then $E$ is a free set with $\operatorname{card} E=\operatorname{dim} V$, but $E$ is not a basis for $V$.

The next result validates the usefulness of the notion of Hamel dimension. 
Theorem 3.8 (Isomorphic Spaces) Let $V$ and $W$ be two vector spaces over the same field $\mathbb{K}$ such that $\operatorname{dim} V=\operatorname{dim} W$. Then $V$ and $W$ are isomorphic. In particular, the mapping $\sigma: V \mapsto W$, defined by $\sigma\left(\sum_{s \in S} c_{s} v_{s}\right)=\sum_{s \in S} c_{s} w_{s}$, is a vector-isomorphism from $V$ to $W$, where $\left(v_{s}\right)_{s \in S}$ and $\left(w_{s}\right)_{s \in S}$ are bases of $V$ and $W$, respectively, $S$ is an index set of $\operatorname{card} S=\operatorname{dim} V=\operatorname{dim} W$ and $c_{s} \in \mathbb{K}$ for all $s \in S$.

Proof The proof is almost identical to the proof of finite-dimensional case and we leave it to the reader.

Lemma 3.9 (Subspace Lemma) Let $U$ and $V$ be two vector spaces over the same field $\mathbb{K}$. Then either $U$ and $V$ are isomorphic, or one of the spaces is (can be embedded as) a subspace of the other (see Remark 3.10). Consequently, if $U$ is a vector subspace of $V$ and $\operatorname{dim} U<\operatorname{dim} V$, then $U$ is a proper subspace of $V$.

Proof Let $\operatorname{dim} U=\alpha$ and $\operatorname{dim} V=\beta$. Then exactly one of the following holds: $\alpha=\beta, \alpha<\beta, \alpha>\beta$, by the axiom of choice in its forth version (Section 2).

Remark 3.10 (Warning) If $U$ and $V$ are infinite-dimensional vector spaces over the same field of scalars $\mathbb{K}$, it might happen that $U$ is a proper subspace of $V$ and at the same time $\operatorname{dim} U=\operatorname{dim} V$. For example, let $\mathbb{K}=\mathbb{R}$ and $V$ be the vector space $\mathbb{R}^{\infty}$ consisting of all sequences in $\mathbb{R}$ with finite support. Let $\left\{\left(e_{1}\right),\left(e_{2}\right), \ldots\right\}$ be the standard basis of $\mathbb{R}^{\infty}$, ie $\left(e_{n}\right)$ is a sequence in $\mathbb{R}$, defined by:

$$
\left(e_{n}\right)_{i}= \begin{cases}1, & \text { if } n=i \\ 0, & \text { if } n \neq i\end{cases}
$$

(For more detail, we refer to Section 6.) Then $U=\operatorname{span}\left\{e_{2}, e_{3}, \ldots\right\}$ is obviously a proper subspace of $\mathbb{R}^{\infty}$. On the other hand, $L \in \mathcal{L}(U, V)$, defined by $L\left(e_{n+1}\right)=e_{n}, n \in$ $\mathbb{N}$, is an isomorphism from $U$ to $V$.

Corollary 3.11 (Algebraic Complement) Every subspace $U$ of $V$ has a (non-unique) algebraic complement $W$ to $V$, ie a subspace $W$ of $V$ such that $V=U \oplus W$.

Proof Let $A \subseteq B$ hold for two (index) sets with $\operatorname{card}(A)=\operatorname{dim}(U)$ and $\operatorname{card} B=\operatorname{dim} V$. Let $\left\{u_{\alpha}: \alpha \in A\right\}$ be a (Hamel) basis of $U$, and

$$
\left\{u_{\alpha}: \alpha \in A\right\} \cup\left\{w_{\beta}: \beta \in B \backslash A\right\}
$$

be its extension to a Hamel basis of $V$ (Theorem 3.6). Then $W=\operatorname{span}\left\{w_{\beta}: \beta \in\right.$ $B \backslash A\}$. 
The next result is in sharp contrast to its counterpart in the finite-dimensional linear algebra.

Lemma 3.12 (Realification) Let $V \mid \mathcal{R}(i)$ denote the vector space $V$ over a field of the form $\mathcal{R}(i)$, where $\mathcal{R}$ is a formally real (orderable) field. Let $V \mid \mathcal{R}$ denote the realification (decomplexification) of $V \mid \mathcal{R}(i)$. Then if one of the vector space is infinite-dimensional, so is the other, and $\operatorname{dim}(V \mid \mathcal{R}(i))=\operatorname{dim}(V \mid \mathcal{R})$.

Proof If $\left\{v_{s}: s \in S\right\}$ is a Hamel basis of $V \mid \mathcal{R}(i)$, then $\left\{v_{s}+i v_{s}: s \in S\right\}$ is a Hamel basis of $V \mid \mathcal{R}$ and $\operatorname{card}\left(\left\{v_{s}: s \in S\right\}\right)=\operatorname{card}\left(\left\{v_{s}+i v_{s}: s \in S\right\}\right)$.

\section{Algebraic dual}

We shortly discuss the properties of the algebraic dual $V^{*}$ of an infinite-dimensional vector space $V$. Both $V^{*}$ and $V^{* *}$ are proper vector space extensions of $V$. In sharp contrast to the finite-dimensional case however, the vector spaces $V, V^{*}$ and $V^{* *}$ are never isomorphic.

Theorem 4.1 (Dimension of Dual Space) Let $V$ be an infinite dimensional vector space over the (infinite) field $\mathbb{K}$ and $V^{*}$ denotes the algebraic dual of $V$. Then $\operatorname{dim} V^{*}=\max \left\{2^{\operatorname{dim} V}, \operatorname{card} \mathbb{K}\right\}=\max \left\{(\operatorname{dim} V)_{+}, \operatorname{card} \mathbb{K}\right\}$. (The formula fails for finite-dimensional $V$.) Consequently, $\operatorname{dim} V^{*}>\operatorname{dim} V$ for any infinite-dimensional vector space $V$.

Proof We start from the formula $\operatorname{dim} V^{*}=(\operatorname{card} \mathbb{K})^{\operatorname{dim} V}$ derived in Jacobson [19, Chapter 9, §5, page 245]. Next, assuming ZFC+GCH (Section 2), we show that $y^{x}=\max \left\{y, 2^{x}\right\}=\max \left\{y, x_{+}\right\}$for every two infinite cardinals, $x$ and $y$. Indeed, if $y=\aleph_{0}$, the formula follows from the fact that $\aleph_{0}<2^{x}$. Let $y$ be uncountable. Then $y=2^{\kappa}$ for some infinite cardinal $\kappa$ by the GCH. Thus:

$$
\begin{aligned}
y^{x} & =\left(2^{\kappa}\right)^{x}=2^{\kappa x}=2^{\max \{\kappa, x\}} \\
& =\left\{\begin{array}{ll}
2^{\kappa}, & \text { if } \kappa \leq x \\
2^{x}, & \text { if } \kappa \geq x
\end{array}= \begin{cases}y, & \text { if } y \leq 2^{x} \\
2^{x}, & \text { if } y \geq 2^{x}\end{cases} \right. \\
& =\max \left\{y, 2^{x}\right\}=\max \left\{y, x_{+}\right\}
\end{aligned}
$$

Finally, we let $y=\operatorname{card}(\mathbb{K})$ and $x=\operatorname{dim} V$. The second equality in the above formula follows from the first equality since $2^{\operatorname{dim} V}=(\operatorname{dim} V)_{+}$by the GCH (Section 2). 
Notice that $\operatorname{dim} V^{*} \geq \operatorname{card} \mathbb{K}$ and $\operatorname{dim} V^{*} \geq(\operatorname{dim} V)_{+}$hold trivially. The next equalities follow immediately from the formula in Theorem 4.1.

Corollary 4.2 (Two More Equalities) (1) If $\operatorname{card} \mathbb{K}<\operatorname{dim} V^{*}$, then $\operatorname{card} V^{*}=$ $\operatorname{dim} V^{*}=(\operatorname{dim} V)_{+} \quad$ (see Example 7.1 in this paper $)$.

(2) If $(\operatorname{dim} V)_{+}<\operatorname{dim} V^{*}$, then $\operatorname{dim} V^{*}=\operatorname{card} \mathbb{K}$ (see Example 7.12).

Definition 4.3 (Embeddings \& Restricted Duals) (1) Let $\mathcal{B}=\left\{v_{s}: s \in S\right\}$ be a basis of $V$ indexed by a set $S$. Let $\left\{\Phi_{r}: r \in S\right\}$ be a subset of $V^{*}$ defined by:

$$
\Phi_{r}\left(v_{s}\right)= \begin{cases}1 & \text { if } r=s \\ 0 & \text { if } r \neq s\end{cases}
$$

The subspace $V_{\mathcal{B}}^{*}=\operatorname{span}\left\{\Phi_{r}: r \in S\right\}$ of $V^{*}$ is the restricted dual of $V$ relative to $\mathcal{B}$. The mapping $\sigma_{\mathcal{B}}: V \mapsto V^{*}$ (with range $\sigma[V]=V_{\mathcal{B}}^{*}$ ), defined by $\sigma_{\mathcal{B}}\left(v_{s}\right)=\Phi_{s}$ for all $s \in S$, is the vector space embedding of $V$ into $V^{*}$ relative to $\mathcal{B}$. We write this as $V \subset_{\mathcal{B}} V^{*}$. If $\mathcal{B}$ is a standard Hamel basis of $V$, we shall write $\sigma, V_{*}$ and $V \subset V^{*}$ instead of $\sigma_{\mathcal{B}}, V_{\mathcal{B}}^{*}$ and $V \subset_{\mathcal{B}} V^{*}$, respectively (for an example we refer to (2) and (3) in Definition 6.1).

(2) The mapping $\iota: V \mapsto V^{* *}$, defined by $\iota(v)(T)=T(v)$ for all $T \in V^{*}$, is called the canonical embedding of $V$ into $V^{* *}$ (it does not depend on a choice of any basis). We write simply, $V \subset V^{* *}$.

Corollary 4.4 (Embedding of Duals) Let $U$ be a subspace of $V$ and $W$ be an algebraic complement of $U$ to $V$, ie $V=U \oplus W$ (Corollary 3.11). Let $\sigma_{W}: U^{*} \mapsto V^{*}$ be the mapping defined by $\sigma_{W}(T)(v)=T(u)$ for all $v \in V$, where $v=u+w, u \in U$ and $w \in W$. Then $\sigma_{W}$ is a vector space embedding of $U^{*}$ into $V^{*}$. We denote this by $U^{*} \subseteq_{W} V^{*}$ or even by $U^{*} \subseteq V^{*}$ (if $W$ is understood).

For a recent study, from a purely algebraic point of view, of the relationship between the restricted dual $V_{*}$ and the algebraic dual $V^{*}$ of a vector space $V$ with a countable Hamel basis, we refer to the recent article Chirvasitu and Penkov [5] (no relation to solvability of PDE and generalized functions).

\section{Linear maps and operators}

We present selected results of linear algebra (needed for the rest of the article) which are well-known for finite-dimensional vector spaces, but less-known for infinite-dimensional spaces. 
Theorem 5.1 (Extension Principle) Let $U, V$ and $W$ be three vector spaces over the same field of scalars, $\mathbb{K}$ (the case $W=\mathbb{K}$ is not excluded) and let $U$ be a subspace of $V$. Then every linear map $L \in \mathcal{L}(U, W)$ can be extended (non-uniquely) to a linear map $\widehat{L} \in \mathcal{L}(V, W)$.

Proof Let $A \subseteq B$ hold for two (index) sets with $\operatorname{card}(A)=\operatorname{dim}(U)$ and $\operatorname{card}(B)=$ $\operatorname{dim}(V)$. Let $\left\{u_{\alpha}: \alpha \in A\right\}$ be a (Hamel) basis of $U$ and $\left\{u_{\alpha}: \alpha \in A\right\} \cup\left\{v_{\beta}: \beta \in B \backslash A\right\}$ be its extension to a (Hamel) basis of $V$ (Theorem 3.6). Let $\widehat{L}: V \mapsto W$ be a map defined by $\widehat{L}\left(u_{\alpha}\right)=L\left(u_{\alpha}\right)$ for $\alpha \in A$ and anything (for example, $\widehat{L}\left(v_{\beta}\right)=0$ ) for $\beta \in B \backslash A$. Then $\widehat{L}$ is an extension of $L$ we are looking for.

Definition 5.2 (Duals and Co-Duals) Let $\mathcal{O} \in \mathcal{L}(V)$ and $\mathcal{O}^{*} \in \mathcal{L}\left(V^{*}\right)$ be two operators such that $\mathcal{O}^{*}(T)=T \circ \mathcal{O}$ for all $T \in V^{*}$. Then we say that the operator $\mathcal{O}^{*}$ is the dual of $\mathcal{O}$ and that $\mathcal{O}$ is the co-dual (or transposed) of $\mathcal{O}^{*}$. We sometimes use the notation $\mathcal{O}={ }^{t}\left(\mathcal{O}^{*}\right)$.

Remarks 5.3 (Bracket Notation) We often write $\langle T, v\rangle$ instead of $T(v)$ for the evaluation of $T \in V^{*}$ at $v \in V$. In this bracket notation the above definition can be summarized as follows: $\left\langle\mathcal{O}^{*}(T), v\right\rangle=\langle T, \mathcal{O}(v)\rangle$ for all $v \in V$ and all $T \in V^{*}$.

Theorem 5.4 (Surjective Dual) Let $V$ be a vector space and $V^{*}$ be its (algebraic) dual. Then $\mathcal{O} \in \mathcal{L}(V)$ is injective iff its dual $\mathcal{O}^{*} \in \mathcal{L}\left(V^{*}\right)$ is surjective.

Proof $(\Rightarrow)$ Let $T \in V^{*}$. We have to show that the equation $\mathcal{O}^{*}(\Lambda)=T$ has a solution $\Lambda$ in $V^{*}$. Indeed, define $\Phi: \operatorname{ran}(\mathcal{O}) \mapsto \mathbb{K}$ by $\Phi(\mathcal{O}(v))=T(v)$ for all $v \in V$, where $\operatorname{ran}(\mathcal{O})$ stands for the range of $\mathcal{O}$. We observe that $\Phi$ is well-defined, because $\mathcal{O}$ is injective by assumption. It is clear that $\Phi \in(\operatorname{ran}(\mathcal{O}))^{*}$, because $T$ is linear. By the Extension Principle (Theorem 5.1), $\Phi$ can be extended to some $\Lambda \in V^{*}$. Thus $\mathcal{O}^{*}(\Lambda)(v)=\Lambda(\mathcal{O}(v))=\Phi(\mathcal{O}(v))=T(v)$ for all $v \in V$. So, $\mathcal{O}^{*}(\Lambda)=T$, as required.

$(\Leftarrow)$ Let $\mathcal{O}(v)=0$ for some $v \in V$. We have to show that $v=0$. Indeed, let $T \in V^{*}$. Since $\mathcal{O}^{*}$ is surjective, there exists $\Lambda \in V^{*}$ such that $T=\mathcal{O}^{*}(\Lambda)$. Thus $T(v)=\mathcal{O}^{*}(\Lambda(v))=\Lambda(\mathcal{O}(v))=\Lambda(0)=0$. Thus $T(v)=0$ for all $T \in V^{*}$ implying $v=0$.

The above result gives rise to the concept of a regular operator (used in Todorov [37] in the particular case of $V=\mathcal{D}\left(\mathbb{R}^{d}\right)$ (Example 7.6). 
Definition 5.5 (Regular Operators) An operator $\mathcal{O}^{*}$ in $\mathcal{L}\left(V^{*}\right)$ is regular if $\mathcal{O}^{*}$ has an injective co-dual (transposed) $\mathcal{O} \in \mathcal{L}(V)$.

Corollary 5.6 (Solvability) Let $V$ be a vector space and $V^{*}$ be its (algebraic) dual. Let $\mathcal{O}^{*} \in \mathcal{L}\left(V^{*}\right)$ be a regular operator. Then the equation $\mathcal{O}^{*}(\Lambda)=T$ is solvable in $V^{*}$ in the sense that for every choice of $T \in V^{*}$ there exists $\Lambda \in V^{*}$ such that $\mathcal{O}^{*}(\Lambda)=T$.

Proof An immediate consequence of Theorem 5.4.

\section{Coordinate isomorphism}

We discuss vector spaces $\mathbb{K}_{0}^{S}$, which are infinite-dimensional counterpart of the familiar vector spaces $\mathbb{K}^{d}$.

Definition 6.1 (The Space $\mathbb{K}_{0}^{S}$ ) Let $\mathbb{K}$ be a field and $S$ be a non-empty set (wellordered if desired).

(1) We denote by $\mathbb{K}_{0}^{S}$ the set of all functions $f: S \mapsto \mathbb{K}$ with finite support $\operatorname{supp}(f)=\{s \in S: f(s) \neq 0\}$.

(2) For each $s \in S$ we define the net $e_{s}: S \mapsto \mathbb{K}$ by

$$
e_{s}(t)= \begin{cases}1, & \text { if } s=t \\ 0, & \text { if } s \neq t\end{cases}
$$

or simply $e_{s}(t)=\delta_{s t}$ for short. We refer to the set $\left\{e_{s}: s \in S\right\}$ as the standard (Hamel) basis of $\mathbb{K}_{0}^{S}$.

(3) Let $\left(\mathbb{K}_{0}^{S}\right)^{*}$ be the (algebraic) dual of $\mathbb{K}_{0}^{S}$ and let $\left\{\Phi_{r}: r \in S\right\}$ be a subset of $\left(\mathbb{K}_{0}^{S}\right)^{*}$ defined by $\Phi_{r}\left(e_{s}\right)=\delta_{r s}$. The subspace $\left(\mathbb{K}_{0}^{S}\right)_{*}=\operatorname{span}\left\{\Phi_{r}: r \in S\right\}$ of $\left(\mathbb{K}_{0}^{S}\right)^{*}$ is the restricted dual of $\mathbb{K}_{0}^{S}$ (Definition 4.3). The mapping $\sigma: \mathbb{K}_{0}^{S} \mapsto\left(\mathbb{K}_{0}^{S}\right)^{*}$, defined by $\sigma\left(e_{s}\right)=\Phi_{s}$ for all $s \in S$, is the vector space embedding of $\mathbb{K}_{0}^{S}$ into $\left(\mathbb{K}_{0}^{S}\right)^{*}$, which will be written simply as $\mathbb{K}_{0}^{S} \subset\left(\mathbb{K}_{0}^{S}\right)^{*}$.

Theorem 6.2 (Properties of $\mathbb{K}_{0}^{S}$ ) Let $\mathbb{K}$ be a field and $S$ be a non-empty set (as above). Then:

(1) $\mathbb{K}_{0}^{S}$ is a vector space over $\mathbb{K}$ and $\left\{e_{s}: s \in S\right\}$ is a bases for $\mathbb{K}_{0}^{S}$. Consequently, $\operatorname{dim} \mathbb{K}_{0}^{S}=\operatorname{card} S$ and $\operatorname{card} \mathbb{K}_{0}^{S}=\max \{\operatorname{card} S, \operatorname{card} \mathbb{K}\}$.

(2) $\operatorname{dim}\left(\mathbb{K}_{0}^{S}\right)^{*}=\operatorname{card}\left(\mathbb{K}_{0}^{S}\right)^{*}=\max \left\{(\operatorname{card} S)_{+}, \operatorname{card} \mathbb{K}\right\}$. 
Proof The part (i) follows immediately from the definition of the spaces $\mathbb{K}_{0}^{S}$ and Lemma 3.3. For part (ii) we calculate: $\operatorname{dim}\left(\mathbb{K}_{0}^{S}\right)^{*}=\max \left\{\left(\operatorname{dim} \mathbb{K}_{0}^{S}\right)_{+}\right.$, card $\left.\mathbb{K}\right\}=$ $\max \left\{(\operatorname{card} S)_{+}, \operatorname{card} \mathbb{K}\right\}$ by Theorem 4.1. Next, we apply Lemma 3.3 in the particular case $V=\left(\mathbb{K}_{0}^{S}\right)^{*}$ and calculate: $\operatorname{card}\left(\mathbb{K}_{0}^{S}\right)^{*}=\max \left\{\operatorname{dim}\left(\mathbb{K}_{0}^{S}\right)^{*}, \operatorname{card} \mathbb{K}\right\}=$ $\max \left\{\left(\max \left\{(\operatorname{card} S)_{+}, \operatorname{card} \mathbb{K}\right\}, \operatorname{card} \mathbb{K}\right\}=\max \left\{(\operatorname{card} S)_{+}, \operatorname{card} \mathbb{K}\right\}\right.$ as required.

Notice that if $S$ is an ordered finite set, the space $\mathbb{K}_{0}^{S}$ reduces to the familiar $\mathbb{K}^{d}$, where $d=\operatorname{card} S$.

Theorem 6.3 (Coordinate Isomorphism) Let $\mathbb{K}$ be a field, $V$ be a vector space over $\mathbb{K}$ (as before). Let $S$ be a set of $\operatorname{card} S=\operatorname{dim} V$ and $\mathcal{B}=\left\{v_{s}: s \in S\right\}$ be a basis of $V$. Then:

(1) The mapping $\Gamma: V \mapsto \mathbb{K}_{0}^{S}$, defined by $\Gamma(v)=f$, where $v=\sum_{s \in S} f(s) v_{s}$, is a vector isomorphism. We call $\Gamma$ a coordinate isomorphism and $f$ the coordinate function of $v$ relative to the basis $\mathcal{B}$ (sometimes the notation $f_{v}$ or even $f_{v, \mathcal{B}}$ should be used instead of $f$ ). In the particular case of $S=\mathcal{B}$, we have $\Gamma: V \mapsto \mathbb{K}_{0}^{\mathcal{B}}$, where $\Gamma(v)=f$ and $v=\sum_{w \in \mathcal{B}} f(w) w$.

(2) For every $s \in S$ we have $\Gamma^{-1}\left(e_{s}\right)=v_{s}$, where $\left\{e_{s}: s \in S\right\}$ is the standard basis of $\mathbb{K}_{0}^{S}$ (Definition 6.1).

Proof (1) We observe that $\Gamma\left(v_{s}\right)=e_{s}$ for every $s \in S$. Thus $\Gamma \in \mathcal{L}\left(V, \mathbb{K}_{0}^{\mathcal{B}}\right)$ is a bijection.

(2) follows directly from (ii).

Remark 6.4 (Well-Ordered Bases) We often use matrices (including row and column matrices) to visualize the coordinate functions of the vectors and linear operators relative to a particular basis of $V$. The matrix approach is exceptionally popular in the cases of finite or countable dimensional vector spaces as well as in separable Hilbert spaces. Can we extend the matrix approach to uncountable vector spaces? The answer is yes; we have to invoke the axiom of choice again in the form of its third version (Section 1): Every set, in particular every basis $\mathcal{B}$ of $V$, can be well-ordered. Alternatively, we can well-order the index set $S$ in $\mathbb{K}_{0}^{S}$. We call these bases well-ordered bases.

\section{Examples of infinite-dimensional spaces}

We present several examples of infinite-dimensional vector spaces and their algebraic duals and demonstrate how to choose an algebraic (Hamel) basis (Theorem 3.6). We 
shall often rely on the formulas:

$$
\begin{aligned}
& \operatorname{card} V=\max \{\operatorname{dim} V, \operatorname{card} \mathbb{K}\} \\
& \operatorname{dim} V^{*}=\max \left\{(\operatorname{dim} V)_{+}, \operatorname{card} \mathbb{K}\right\} \\
& \operatorname{card} V^{*}=\max \left\{\operatorname{dim} V^{*}, \operatorname{card} \mathbb{K}\right\}
\end{aligned}
$$

(Lemma 3.3 and Theorem 4.1) along with the shortcuts presented in Corollary 3.5 and Corollary 4.2. Here is some advice for the order of the calculations. If $\operatorname{dim}(V)$ is known (or easy to calculate), we recommend the order of calculations: $\operatorname{dim}(V) \mapsto \operatorname{card}(V) \mapsto$ $\operatorname{dim}\left(V^{*}\right) \mapsto \operatorname{card}\left(V^{*}\right)$. If $\operatorname{card}(V)$ is known (or easy to calculate), we recommend: $\operatorname{card}(V) \mapsto \operatorname{dim}(V) \mapsto \operatorname{dim}\left(V^{*}\right) \mapsto \operatorname{card}\left(V^{*}\right)$. Recall that $\operatorname{dim}(V) \leq \operatorname{card}(V)$ holds trivially.

Example 7.1 (Hamel Example) Let $\mathbb{R} \mid \mathbb{Q}$ denote the $\mathbb{Q}$-vector space of $\mathbb{R}$ and $(\mathbb{R} \mid \mathbb{Q})^{*}$ stand for its (algebraic) dual. We have $\operatorname{dim}(\mathbb{R} \mid \mathbb{Q})=\operatorname{card} \mathbb{R}=\mathfrak{c}$ by Corollary 3.5 , since $\operatorname{card} \mathbb{Q}<\operatorname{card} \mathbb{R}$. Also, $\operatorname{dim}(\mathbb{R} \mid \mathbb{Q})^{*}=\max \left\{\mathfrak{c}_{+}, \aleph_{0}\right\}=\mathfrak{c}_{+}$by $(2)$ and $\operatorname{card}(\mathbb{R} \mid \mathbb{Q})^{*}=\mathfrak{c}_{+}$ by Corollary 4.2, since again, card $\mathbb{Q}<$ card $\mathbb{R}$. For the original source of this example, we refer to Hamel [12].

Example 7.2 (The Space $\mathbb{R} \mid \mathcal{A}$ and its Dual) Let $\mathcal{A}$ denote the field of algebraic real numbers. Let $\mathbb{R} \mid \mathcal{A}$ denote the $\mathcal{A}$-vector space of $\mathbb{R}$ and $(\mathbb{R} \mid \mathcal{A})^{*}$ stand for its dual. As in the previous example, we have $\operatorname{dim}(\mathbb{R} \mid \mathcal{A})=\operatorname{card} \mathbb{R}=\mathfrak{c}$ and $\operatorname{dim}(\mathbb{R} \mid \mathbb{Q})^{*}=$ $\operatorname{card}(\mathbb{R} \mid \mathcal{A})^{*}=\mathfrak{c}_{+}$since $\operatorname{card} \mathcal{A}=\operatorname{card} \mathbb{Q}<\operatorname{card} \mathbb{R}$.

Example 7.3 $\left(\mathbb{R}^{\mathbb{N}}\right.$ and its Dual) Let $\mathbb{R}^{\mathbb{N}}$ denote the $\mathbb{R}$-vector space of all sequences in $\mathbb{R}$ and $\left(\mathbb{R}^{\mathbb{N}}\right)^{*}$ denote its dual space. We show that $\operatorname{dim}\left(\mathbb{R}^{\mathbb{N}}\right)=\operatorname{card}\left(\mathbb{R}^{\mathbb{N}}\right)=\mathfrak{c}$ and $\operatorname{dim}\left(\mathbb{R}^{\mathbb{N}}\right)^{*}=\operatorname{card}\left(\mathbb{R}^{\mathbb{N}}\right)^{*}=\mathfrak{c}_{+} . \operatorname{Indeed}, \operatorname{card}\left(\mathbb{R}^{\mathbb{N}}\right)=\mathfrak{c}^{\aleph_{0}}=\left(2^{\aleph_{0}}\right)^{\aleph_{0}}=2^{\aleph_{0} \aleph_{0}}=2^{\aleph_{0}}=\mathfrak{c}$ (the last step is due to the $\mathrm{CH}$, Section 2). On the other hand, $\operatorname{dim}\left(\mathbb{R}^{\mathbb{N}}\right) \leq \mathfrak{c}$ holds trivially. To show $\operatorname{dim}\left(\mathbb{R}^{\mathbb{N}}\right) \geq \mathfrak{c}$, observe that the subset $E=\left\{\left(\frac{1}{r^{n}}\right): r \in \mathbb{R}, r \neq 0\right\}$ of $\mathbb{R}^{\mathbb{N}}$ is a free set of $\mathbb{R}^{\mathbb{N}}$, where $\left(\frac{1}{r^{n}}\right)=\left(\frac{1}{r}, \frac{1}{r^{2}}, \ldots\right)$. Thus $\operatorname{dim}\left(\mathbb{R}^{\mathbb{N}}\right)=\mathfrak{c}$ (as required) and $\mathbb{R}^{\mathbb{N}} \cong \mathbb{R}_{0}^{\mathbb{R}^{2}}$ by Theorem 6.3, since card $\left(\mathbb{R}^{2}\right)=\mathfrak{c}$. Consequently, $E$ can be extended to a Hamel basis, say, $\mathcal{B}=\left\{g_{(r, s)}:(r, s) \in \mathbb{R}^{2},(r, s) \neq(0,0)\right\}$ of $\mathbb{R}^{\mathbb{N}}$ by Theorem 3.6, where $g_{(r, 0)}=\left(\frac{1}{r^{n}}\right)$ for all $r \in \mathbb{R}, r \neq 0$.

Next, $\operatorname{dim}\left(\mathbb{R}^{\mathbb{N}}\right)^{*}=\max \left\{\mathfrak{c}_{+}, \mathfrak{c}\right\}=c_{+}$by (2) and card $\left(\mathbb{R}^{\mathbb{N}}\right)^{*}=\mathfrak{c}_{+}$by Corollary 4.2, since card $\mathbb{R}<\operatorname{dim}\left(\mathbb{R}^{\mathbb{N}}\right)^{*}$. Consequently, $\left(\mathbb{R}^{\mathbb{N}}\right)^{*} \cong \mathbb{R}_{0}^{\mathcal{P}\left(\mathbb{R}^{2}\right)}$ by Theorem 6.3. Let us consider the subset

$$
E^{*}=\left\{\varepsilon_{\{(a, b)\}}:(a, b) \in \mathbb{R}^{2},(a, b) \neq(0,0)\right\}
$$


of $\left(\mathbb{R}^{\mathbb{N}}\right)^{*}$, defined by:

$$
\varepsilon_{\{(a, b)\}}\left(g_{(r, s)}\right)= \begin{cases}1, & \text { if }(a, b)=(r, s) \\ 0, & \text { otherwise }\end{cases}
$$

We observe that $E^{*}$ is a free set of $\left(\mathbb{R}^{\mathbb{N}}\right)^{*}$. Thus $E^{*}$ can be extended to a basis $\mathcal{B}^{*}=\left\{\varepsilon_{A}\right.$ : $\left.A \in \mathcal{P}\left(\mathbb{R}^{2}\right)\right\}$ of $\left(\mathbb{R}^{\mathbb{N}}\right)^{*}$ by Theorem 3.6, where $\varepsilon_{A}=\varepsilon_{\{(a, b)\}}$ for all $A=\{(a, b)\}$ such that $(a, b) \neq(0,0)$. The subspace $\left(\mathbb{R}^{\mathbb{N}}\right)_{\mathcal{B}}^{*}=\operatorname{span}\left\{\varepsilon_{\{(a, b)\}}:(a, b) \in \mathbb{R}^{2},(a, b) \neq(0,0)\right\}$ of $\left(\mathbb{R}^{\mathbb{N}}\right)^{*}$ is the restricted dual of $\mathbb{R}^{\mathbb{N}}$ relative to the base $\mathcal{B}$ (Definition 4.3).

Example 7.4 $\left(\mathbb{C}^{\mathbb{N}}\right.$ and its Dual) Let $\mathbb{C}^{\mathbb{N}}$ denote the $\mathbb{C}$-vector space of all sequences in $\mathbb{C}$ and let $\left(\mathbb{C}^{\mathbb{N}}\right)^{*}$ be its algebraic dual. Similarly to the previous example, we have $\operatorname{dim}\left(\mathbb{C}^{\mathbb{N}}\right)=\operatorname{card}\left(\mathbb{C}^{\mathbb{N}}\right)=\mathfrak{c}$ and $\operatorname{dim}\left(\mathbb{C}^{\mathbb{N}}\right)^{*}=\operatorname{card}\left(\mathbb{C}^{\mathbb{N}}\right)^{*}=\mathfrak{c}_{+}$Consequently, $\mathbb{C}^{\mathbb{N}} \cong \mathbb{C}_{0}^{\mathbb{R}^{2}}$ and $\left(\mathbb{C}^{\mathbb{N}}\right)^{*} \cong \mathbb{C}_{0}^{\mathcal{P}\left(\mathbb{R}^{2}\right)}$.

Example 7.5 (The Space of Polynomials and its Dual) Let $\mathbb{C}[z]$ denote the $\mathbb{C}$-vector space consisting of all polynomials with coefficients in $\mathbb{C}$. Let $(\mathbb{C}[z])^{*}$ denote the algebraic dual of $\mathbb{C}[z]$.

We have $\operatorname{dim}(\mathbb{C}[z])=\aleph_{0}$, because $\left(1, z, z^{2}, \ldots\right)$ is (obviously) a basis of $\mathbb{C}[z]$. Also, $\operatorname{card} \mathbb{C}[z]=\max \{\operatorname{dim} \mathbb{C}[z], \operatorname{card} \mathbb{C}\}=\max \left\{\aleph_{0}, \mathfrak{c}\right\}=\mathfrak{c}$ by $(1)$.

Next, $\operatorname{dim}(\mathbb{C}[z])^{*}=\max \left\{\left(\aleph_{0}\right)_{+}, \operatorname{card} \mathbb{C}\right\}=\max \{\mathfrak{c}, \mathfrak{c}\}=\mathfrak{c}$ by $(2)$ and $\operatorname{card}(\mathbb{C}[z])^{*}=$ $\max \left\{\operatorname{dim}(\mathbb{C}[z])^{*}, \operatorname{card} \mathbb{C}\right\}=\max \{\mathfrak{c}, \mathfrak{c}\}=\mathfrak{c}$ by (3). Thus, $\operatorname{dim} \mathbb{C}[z]=\aleph_{0}$ and $\operatorname{card} \mathbb{C}[z]=\operatorname{dim}(\mathbb{C}[z])^{*}=\operatorname{card}(\mathbb{C}[z])^{*}=\mathfrak{c}$.

The next example is important for the rest of the article.

Example 7.6 $\left(\mathcal{D}(\Omega)\right.$ and its Dual) Let $\Omega$ be an open set of $\mathbb{R}^{d}$ and $\mathcal{D}(\Omega)=\mathcal{C}_{0}^{\infty}(\Omega)$ denote the space of test-functions on $\Omega$ (Vladimirov [39]). Let $\mathcal{D}^{*}(\Omega)$ denote the algebraic dual of $\mathcal{D}(\Omega)$ (not to be confused with the space of Schwartz distributions $\mathcal{D}^{\prime}(\Omega)$, Vladimirov [39]).

We show that $\operatorname{dim} \mathcal{D}(\Omega)=\operatorname{card} \mathcal{D}(\Omega)=\mathfrak{c}$. Indeed, $\operatorname{card} \mathcal{D}(\Omega) \leq \mathfrak{c}$, because the mapping $\psi: \mathcal{D}(\Omega) \mapsto \mathbb{C}^{\Omega \cap \mathbb{Q}^{d}}, \psi(\varphi)=\varphi \mid \Omega \cap \mathbb{Q}^{d}$, is an injection (due to the continuity of $\varphi)$ and $\operatorname{card}\left(\mathbb{C}^{\Omega \cap \mathbb{Q}^{d}}\right)=2^{\aleph_{0}}=\mathfrak{c}$. Thus $\operatorname{dim} \mathcal{D}(\Omega) \leq \mathfrak{c}$ since $\operatorname{dim} \mathcal{D}(\Omega) \leq \operatorname{card} \mathcal{D}(\Omega)$ holds trivially. To show that $\operatorname{dim} \mathcal{D}(\Omega) \geq \mathfrak{c}$, we observe that the set $E=\left\{\tau_{h} \varphi \in \mathcal{D}(\Omega)\right.$ : $\left.h \in \mathbb{R}^{d}\right\}$, is a free set of $\mathcal{D}(\Omega)$ (Definition 3.1). Here $\varphi \in \mathcal{D}(\Omega)$ is a (fixed) non-zero test-function and $\left(\tau_{h} \varphi\right)(x)=\varphi(x-h)$. Indeed, suppose $\sum_{n=1}^{m} c_{n} \tau_{h_{n}} \varphi=0$ for some $m \in \mathbb{N}$, some $c_{n} \in \mathbb{C}$ and some mutually distinct $h_{n} \in \mathbb{R}^{d}$ such that $\tau_{h_{n}} \varphi \in \mathcal{D}(\Omega)$. The Fourier transform produces $\left(\sum_{n=1}^{m} c_{n} e^{-i h_{n} z}\right) \mathcal{F}[\varphi](z)=0$, where both $\sum_{n=1}^{m} c_{n} e^{-i h_{n} z}$ 
and $\mathcal{F}[\varphi](z)$ are entire functions. So, we can cancel $\mathcal{F}[\varphi](z)$, because the ring of entire functions forms an integral domain. However, $\sum_{n=1}^{m} c_{n} e^{-i h_{n} z}=0$ implies $c_{1}=\cdots=c_{m}=0$ (as desired), since the exponents are linearly independent. Thus $\operatorname{dim} \mathcal{D}(\Omega)=\mathfrak{c}$. Finally, $\operatorname{card} \mathcal{D}(\Omega)=\max \{\operatorname{dim} \mathcal{D}(\Omega), \operatorname{card} \mathbb{C}\}=\max \{\mathfrak{c}, \mathfrak{c}\}=\mathfrak{c}$ (as required) by (1). Consequently, $\mathcal{D}(\Omega) \cong \mathbb{C}_{0}^{\mathbb{R}^{d} \times \mathbb{R}}$, since card $\left(\mathbb{R}^{d} \times \mathbb{R}\right)=\mathfrak{c}$ (Section 6). Next, the free set $E$ can be extended to a basis $\mathcal{B}_{\mathcal{D}(\Omega)}$ of $\mathcal{D}(\Omega)$ of the form $\mathcal{B}_{\mathcal{D}(\Omega)}=\left\{\varphi_{h, r}:(h, r) \in \mathbb{R}^{d} \times \mathbb{R}\right\}$, where $\varphi_{h, 0}=\tau_{h} \varphi$ for all $h \in \mathbb{R}^{d}$ such that $\tau_{h} \varphi \in \mathcal{D}(\Omega)$ (Theorem 3.6).

We show that $\operatorname{dim} \mathcal{D}^{*}(\Omega)=\operatorname{card} \mathcal{D}^{*}(\Omega)=\mathfrak{c}_{+}$. Indeed,

$$
\operatorname{dim} \mathcal{D}^{*}(\Omega)=\max \left\{(\operatorname{dim} \mathcal{D}(\Omega))_{+}, \operatorname{card} \mathbb{C}\right\}=\max \left\{\mathfrak{c}_{+}, \mathfrak{c}\right\}=\mathfrak{c}_{+}
$$

by (2). Also, we observe that $\operatorname{card} \mathcal{D}^{*}(\Omega)=\mathfrak{c}_{+}$by Corollary 4.2, since card $\mathbb{C}<$ $\operatorname{dim} \mathcal{D}^{*}(\Omega)$. Thus $\mathcal{D}^{*}(\Omega) \cong \mathbb{C}_{0}^{\mathcal{P}\left(\mathbb{R}^{d} \times \mathbb{R}\right)}$, since $\operatorname{card} \mathcal{P}\left(\mathbb{R}^{d} \times \mathbb{R}\right)=\mathfrak{c}_{+}$(Section 6).

Here is one particular choice of a basis $\mathcal{B}_{\mathcal{D}^{*}(\Omega)}$ of $\mathcal{D}^{*}(\Omega)$. Let $\left\{\Phi_{g, s}:(g, s) \in \mathbb{R}^{d} \times \mathbb{R}\right\}$ be the subset of $\mathcal{D}^{*}(\Omega)$ such that:

$$
\Phi_{g, s}\left(\varphi_{h, r}\right)= \begin{cases}1, & \text { if }(g, s)=(h, r) \\ 0, & \text { if }(g, s) \neq(h, r)\end{cases}
$$

It is clear that $\left\{\Phi_{g, s}:(g, s) \in \mathbb{R}^{d} \times \mathbb{R}\right\}$ is a free set of $\mathcal{D}^{*}(\Omega)$ and thus, it can be extended to a basis $\mathcal{B}_{\mathcal{D}^{*}(\Omega)}=\left\{\Phi_{X}: X \in \mathcal{P}\left(\mathbb{R}^{d} \times \mathbb{R}\right)\right\}$ of $\mathcal{D}^{*}(\Omega)$ by Theorem 3.6, where $\Phi_{\{(h, r)\}}=\Phi_{h, r}$ for all $(h, r) \in \mathbb{R}^{d} \times \mathbb{R}$. The subspace $\mathcal{D}^{*}(\Omega)_{\mathcal{B}_{\mathcal{D}(\Omega)}}=\operatorname{span}\left\{\Phi_{h, r}\right.$ : $\left.(h, r) \in \mathbb{R}^{d} \times \mathbb{R}\right\}$ of $\mathcal{D}^{*}(\Omega)$ is the restricted dual of $\mathcal{D}(\Omega)$ relative to the base $\mathcal{B}_{\mathcal{D}(\Omega)}$ (Definition 4.3).

Example 7.7 $\left(\mathcal{E}(\Omega)\right.$ and its Dual) Let $\mathcal{E}(\Omega)=\mathcal{C}^{\infty}(\Omega)$ and let $\mathcal{E}^{*}(\Omega)$ denote the algebraic dual of $\mathcal{E}(\Omega)$. Similarly to the previous example, we have card $\mathcal{E}(\Omega)=$ $\operatorname{dim} \mathcal{E}(\Omega)=\mathfrak{c}$ and $\operatorname{card} \mathcal{E}^{*}(\Omega)=\operatorname{dim} \mathcal{E}^{*}(\Omega)=\mathfrak{c}_{+}$. Thus $\mathcal{E}(\Omega) \cong \mathbb{C}_{0}^{\mathbb{R}^{d} \times \mathbb{R}}$ and $\mathcal{E}^{*}(\Omega) \cong \mathbb{C}_{0}^{\mathcal{P}\left(\mathbb{R}^{d} \times \mathbb{R}\right)}($ Section 6$)$. Notice that $\mathcal{D}^{*}(\Omega) \subset \mathcal{E}^{*}(\Omega)$ by Corollary 4.4 , in sharp contrast to the embedding of the distributions with compact support $\mathcal{E}^{\prime}(\Omega) \subset \mathcal{D}^{\prime}(\Omega)$ in distribution theory (Vladimirov [39, page 43]).

Example 7.8 $\left(\mathcal{D}^{\prime}(\Omega)\right.$ and its Dual) Let $\mathcal{D}^{\prime}(\Omega)$ denote the space of Schwartz distributions on $\Omega$ (Vladimirov [39]) and let $\mathcal{D}^{* *}(\Omega)$ denote the algebraic dual of $\mathcal{D}^{\prime}(\Omega)$.

We have card $\mathcal{D}^{\prime}(\Omega)=\operatorname{dim} \mathcal{D}^{\prime}(\Omega)=\mathfrak{c}$, because $\mathcal{D}^{\prime}(\Omega)$ is sequentially separable in the weak-star-topology (the topology of the pointwise convergence, Definition 8.1) and also, $\mathcal{D}^{\prime}(\Omega)$ is a countable union of weak-star-bounded sets of $\mathcal{D}^{\prime}(\Omega)$; the polars of the elements of a countable subsets of $\mathcal{D}(\Omega)$. It remains to take into account that the 
weak-star-bounded sets of $\mathcal{D}^{\prime}(\Omega)$ are metrizable in the weak-star-topology; thus, of cardinality at most $\mathfrak{c}$ (Köthe $[22$, page $261, \S 21.3(4)]$ ). Consequently, $\mathcal{D}^{\prime}(\Omega)$ and $\mathbb{C}_{0}^{\mathbb{R}^{d} \times \mathbb{R}}$ are isomorphic $\mathbb{C}$-vector spaces, since $\operatorname{card}\left(\mathbb{R}^{d} \times \mathbb{R}\right)=\mathfrak{c}$.

Next, we have card $\mathcal{D}^{\prime *}(\Omega)=\operatorname{dim} \mathcal{D}^{\prime *}(\Omega)=\mathfrak{c}_{+}$. Indeed, $\operatorname{dim} \mathcal{D}^{\prime *}(\Omega)=\max \left\{\mathfrak{c}_{+}\right.$, card $\mathbb{C}\}=\mathfrak{c}_{+}$by (2) and card $\mathcal{D}^{\prime *}(\Omega)=\max \left\{\mathfrak{c}_{+}, \mathfrak{c}\right\}=\mathfrak{c}_{+}$by (3). Consequently, $\mathcal{D}^{\prime *}(\Omega)$ and $\mathbb{C}_{0}^{\mathcal{P}\left(\mathbb{R}^{d} \times \mathbb{R}\right)}$ are isomorphic $\mathbb{C}$-vector spaces (Section 6).

Example 7.9 (Hilbert Space $\mathcal{H}$ and its Dual) Let $\mathcal{H}$ be a separable (infinitedimensional) Hilbert space over $\mathbb{C}$ with an inner product $\langle\cdot, \cdot\rangle$. Let $\left(e_{1}, e_{2}, \ldots\right)$ be a Hilbert (not Hamel) basis of $\mathcal{H}$. Recall that $H=\operatorname{span}\left\{e_{1}, e_{2}, \ldots\right\}$ is a proper inner subspace of $\mathcal{H}$, which is dense in $\mathcal{H}$ relative to the norm topology generated by the norm $\sqrt{\langle\cdot, \cdot\rangle}$. Let $\mathcal{H}^{*}$ denote the algebraic dual of $\mathcal{H}$ and $\mathcal{H}^{* *}$ denote the algebraic double dual of $\mathcal{H}$. We have card $\mathcal{H}=\operatorname{dim} \mathcal{H}=\mathfrak{c}$ and thus $\mathcal{H} \cong \mathbb{C}_{0}^{\mathbb{R}}$. Indeed, card $\mathcal{H} \geq \mathfrak{c}$ by (1), since card $\mathbb{C}=\mathfrak{c}$. The inequality card $\mathcal{H} \leq \mathfrak{c}$ follows from fact that $\mathcal{H}$ is a metric space (hence, of cardinality at most $\mathfrak{c}$ ). Thus, $\operatorname{card} \mathcal{H}=\mathfrak{c}$. Next, $\operatorname{dim} \mathcal{H} \leq \operatorname{card} \mathcal{H}=\mathfrak{c}$ holds trivially. To show $\operatorname{dim} \mathcal{H} \geq \mathfrak{c}$, observe that $\operatorname{dim}(\mathcal{H})=\operatorname{dim}\left(\mathcal{L}^{2}(\mathbb{R})\right)$, because $\mathcal{H}$ and $\mathcal{L}^{2}(\mathbb{R})$ are vector-isomorphic (as separable Hilbert spaces), $\mathcal{D}(\mathbb{R}) \subset \mathcal{L}^{2}(\mathbb{R})$ and $\operatorname{dim} \mathcal{D}(\mathbb{R})=\mathfrak{c}($ Example 7.6). Thus, $\operatorname{dim} \mathcal{H}=\mathfrak{c}$ as required.

How do we choose a Hamel basis of $\mathcal{H}$ ? Unfortunately, Hilbert spaces (separable or not) do not have orthonormal Hamel bases. Suppose (seeking a contradiction) that $\mathcal{B}$ is an orthonormal Hamel basis of a Hilbert space $\mathcal{H}$. Let $\left(e_{1}, e_{2}, \ldots\right)$ be an orthonormal sequence in $\mathcal{H}$. Consider the sequence $\left(v_{1}, v_{2}, \ldots\right)$ in $\mathcal{H}$ by $v_{1}=e_{1}, v_{2}=e_{1}+\frac{1}{2} e_{2}$, etc $v_{n}=\sum_{k=1}^{n} \frac{e_{k}}{k}$. Then $\left(v_{1}, v_{2}, \ldots\right)$ is a Cauchy sequence, but it is divergent. Indeed, suppose (seeking a contradiction again) that $\lim _{n \mapsto \infty}\left\|v_{n}-v\right\|$ for some $v \in \mathcal{H}$. We have $v=\sum_{k=1}^{m} c_{k} w_{k}$ for some $m \in \mathbb{N}$, some $c_{k} \in \mathbb{C}$ and some $w_{k} \in \mathcal{B}$. After replacing, we get $\lim _{n \mapsto \infty}\left\|\sum_{k=1}^{n} \frac{e_{k}}{k}-\sum_{k=1}^{m} c_{k} w_{k}\right\|=0$, a contradiction, since in $\left(w_{1}, \cdots, w_{m}, e_{1}, e_{2}, \ldots\right)$ there are not more than finitely many repetitions and the set $\left\{w_{1}, \cdots, w_{m}, e_{1}, e_{2}, \ldots\right\}$ consists of mutually orthogonal unit vectors only. Thus, $\mathcal{H}$ is non-complete, another contradiction.

So, we have to choose a non-orthonormal Hamel basis of $\mathcal{H}$, which is desirable to be as close to an orthonormal as possible. One way to do this is to start from a Hilbert (non-Hamel) orthonormal basis $\left(e_{1}, e_{2}, \ldots\right)$ of $\mathcal{H}$ (mentioned already above) and to extend it (non-uniquely) to a Hamel basis $\mathcal{B}_{\mathcal{H}}=\left\{e_{r}: r \in \mathbb{R}\right\}$ of $\mathcal{H}$, by Theorem 3.6. Note that the basis $\mathcal{B}_{\mathcal{H}}$ is non-orthogonal (although it is an extension of an orthonormal Hilbert basis).

We show now that card $\mathcal{H}^{*}=\operatorname{dim} \mathcal{H}^{*}=\mathfrak{c}_{+}$and thus $\mathcal{H}^{*} \cong \mathbb{C}_{0}^{\mathcal{P}(\mathbb{R})}($ Section 6). Indeed, we have $\operatorname{dim}\left(\mathcal{H}^{*}\right)=\max \left\{(\operatorname{dim} V)_{+}, \operatorname{card} \mathbb{C}\right\}=\max \left\{\mathfrak{c}_{+}, \mathfrak{c}\right\}=\mathfrak{c}_{+}$by (2). Also, 
$\operatorname{card}\left(\mathcal{H}^{*}\right)=\max \left\{\operatorname{dim} \mathcal{H}^{*}, \operatorname{card} \mathbb{C}\right\}=\max \left\{\mathfrak{c}_{+}, \mathfrak{c}\right\}=\mathfrak{c}_{+}$by (3). Let $\left\{\varepsilon_{s}: s \in \mathbb{R}\right\}$ be the subset of $\mathcal{H}^{*}$ defined by $\varepsilon_{s}\left(e_{r}\right)=\delta_{s r}$ for all $r, s \in \mathbb{R}$. It is clear that $\left\{\varepsilon_{s}: s \in \mathbb{R}\right\}$ is a free set of $\mathcal{H}^{*}$ and thus it can be extended to a basis $\mathcal{B}_{\mathcal{H}^{*}}=\left\{\varepsilon_{s}: s \in \mathcal{P}(\mathbb{R})\right\}$ of $\mathcal{H}^{*}$. The subspace $\mathcal{H}_{\mathcal{B}_{\mathcal{H}}}^{*}=\operatorname{span}\left\{\varepsilon_{s}: s \in \mathbb{R}\right\}$ of $\mathcal{H}^{*}$ is the restricted dual of $\mathcal{H}$ relative to $\mathcal{B}_{\mathcal{H}}$ (Definition 4.3).

Finally, we have $\operatorname{dim} \mathcal{H}^{* *}=\operatorname{card} \mathcal{H}^{* *}=\left(\mathfrak{c}_{+}\right)_{+}$by (1)-(3) and thus $\mathcal{H}^{* *} \cong \mathbb{C}_{0}^{\mathcal{P}(\mathcal{P}(\mathbb{R}))}$ (Section 6). Let $\iota: \mathcal{H} \mapsto \mathcal{H}^{* *}$ be the canonical embedding of $\mathcal{H}$ into $\mathcal{H}^{* *}$, defined by $\iota(\varphi)(T)=T(\varphi)$ for all $T \in \mathcal{H}^{*}$ (Definition 4.3). Then $\iota\left[\mathcal{B}_{\mathcal{H}}\right]$ is a Hamel basis of $\iota[\mathcal{H}]$. Let $W$ be an algebraic complement of $\iota[\mathcal{H}]$ to $\mathcal{H}^{* *}$, ie $\mathcal{H}^{* *}=\iota[\mathcal{H}] \oplus W$ (Corollary 3.11). Let $\mathcal{B}_{W}=\left\{w_{A}: A \in \mathcal{P}(\mathcal{P}(\mathbb{R})) \backslash \mathbb{R}\right\}$ be a Hamel basis of $W$. Then $\mathcal{B}^{* *}=\iota\left[\mathcal{B}_{\mathcal{H}}\right] \cup \mathcal{B}_{W}$ is a Hamel basis of $\mathcal{H}^{* *}$.

Remark 7.10 (An Alternative) Alternatively to the example above, we can define a (new) inner product on $\mathcal{H}$ by $(v, w)=\sum_{r \in \operatorname{sp}(v) \cap \operatorname{sp}(w)} \bar{a}_{r} b_{r}$, where $v=\sum_{r \in \operatorname{sp}(v)} a_{r} e_{r} \in$ $\mathcal{H}$ and $w=\sum_{s \in \operatorname{sp}(w)} b_{s} e_{s} \in \mathcal{H}$ (Definition 3.4). We observe that $\operatorname{sp}\left(e_{r}\right)=\{r\}$. Thus, $\left(e_{r}, e_{s}\right)=\delta_{r s}$ for all $r, s \in \mathbb{R}$. The latter means that $\mathcal{B}_{\mathcal{H}}=\left\{e_{r}: r \in \mathbb{R}\right\}$ is an orthonormal Hamel basis of $\mathcal{H}$ relative to $(\cdot, \cdot)$. We observe that $(\cdot, \cdot)$ coincides with $\langle\cdot, \cdot\rangle$ on $H=\operatorname{span}\left\{e_{1}, e_{2}, \ldots\right\}$, since $\mathcal{B}_{\mathcal{H}}=\left\{e_{r}: r \in \mathbb{R}\right\}$ is an extension of $\left\{e_{1}, e_{2}, \ldots\right\}$. Notice however, that $(\mathcal{H},(\cdot, \cdot))$ is not a Hilbert space by what was explained above; it is non-complete relative to the norm $\sqrt{(\cdot, \cdot)}$. Rather, $(\mathcal{H},(\cdot, \cdot))$ is merely an infinite-dimensional inner vector space over $\mathbb{C}$, which admits an orthonormal Hamel basis and which shares with $(\mathcal{H},\langle\cdot, \cdot\rangle)$ a common inner subspace $H$.

Summarizing, the vector spaces $\mathcal{D}(\Omega), \mathcal{E}(\Omega), \mathcal{D}^{\prime}(\Omega), \mathcal{E}^{\prime}(\Omega), \mathcal{L}^{2}(\Omega), l_{2}(\mathbb{C}), \mathcal{H}, \mathbb{C}_{0}^{\mathbb{R}^{d} \times \mathbb{R}}$ and $\mathbb{C}_{0}^{\mathbb{R}}$ are mutually isomorphic and all of dimension $\mathfrak{c}$. Also, $\mathcal{D}^{*}(\Omega), \mathcal{D}^{\prime *}(\Omega), \mathcal{E}^{*}(\Omega)$, $\left(\mathcal{L}^{2}(\Omega)\right)^{*},\left(l_{2}(\mathbb{C})\right)^{*}, \mathcal{H}^{*}, \mathbb{C}_{0}^{\mathcal{P}\left(\mathbb{R}^{d} \times \mathbb{R}\right)}$ and $\mathbb{C}_{0}^{\mathcal{P}(\mathbb{R})}$ are mutually isomorphic and all of dimension $\mathfrak{c}_{+}$. Similarly, $\mathcal{D}^{* *}(\Omega), \mathcal{D}^{\prime * *}(\Omega), \mathcal{E}^{* *}(\Omega),\left(\mathcal{L}^{2}(\Omega)\right)^{* *},\left(l_{2}(\mathbb{C})\right)^{* *}, \mathcal{H}^{* *}$ and $\mathbb{C}_{0}^{\mathcal{P}(\mathcal{P}(\mathbb{R}))}$ are mutually isomorphic and all of dimension $\left(\mathfrak{c}_{+}\right)_{+}$.

The next several examples are about vector spaces over a field of nonstandard complex numbers $* \mathbb{C}$. The reader who is unfamiliar with nonstandard analysis (Robinson [33]), might skip these examples and resume the reading from Section 8. Recall that for every infinite cardinal $\kappa$ there exists a unique (up to a field isomorphism) nonstandard extension ${ }^{*} \mathbb{C}$ of $\mathbb{C}$ in a $\kappa_{+}$-saturated ultrapower nonstandard model with the set of individuals $\mathbb{R}$ (Chang and Keisler [4]; for a presentation we refer also to the Appendix in Lindstrøm [25]). Recall as well that $* \mathbb{C}$ is an algebraically closed non-Archimedean field containing $\mathbb{C}$ (as a subfield) with $\operatorname{card}\left({ }^{*} \mathbb{C}\right)=\kappa_{+}$. Also, ${ }^{*} \mathbb{C}={ }^{*} \mathbb{R}(i)$, where ${ }^{*} \mathbb{R}$ is a $\kappa_{+}-$saturated real closed non-Archimedean field containing $\mathbb{R}$ as a subfield. We 
should alert the reader that the asterisk in front, ${ }^{*} \mathbb{C}$, has nothing to do with the asterisk after, $\mathbb{C}^{*}$.

Example 7.11 (The Space ${ }^{*} \mathbb{C} \mid \mathbb{C}$ and its Dual) Let $* \mathbb{C} \mid \mathbb{C}$ denote the $\mathbb{C}$-vector space of $* \mathbb{C}$ and $\left({ }^{*} \mathbb{C} \mid \mathbb{C}\right)^{*}$ stand for its dual. We have $\operatorname{card}\left({ }^{*} \mathbb{C}\right)=\operatorname{card}\left({ }^{*} \mathbb{C} \mid \mathbb{C}\right)=$ $\operatorname{dim}\left({ }^{*} \mathbb{C} \mid \mathbb{C}\right)=\kappa_{+}$and $\operatorname{card}\left({ }^{*} \mathbb{C} \mid \mathbb{C}\right)^{*}=\operatorname{dim}\left({ }^{*} \mathbb{C} \mid \mathbb{C}\right)^{*}=\left(\kappa_{+}\right)_{+}$. Indeed:

- If $\kappa>\aleph_{0}$, then $\operatorname{dim}\left({ }^{*} \mathbb{C} \mid \mathbb{C}\right)=\kappa_{+}$by Corollary 3.5, since card $\mathbb{C}<\operatorname{card}\left({ }^{*} \mathbb{C}\right)$. Next, $\operatorname{dim}\left({ }^{*} \mathbb{C} \mid \mathbb{C}\right)^{*}=\max \left\{\left(\kappa_{+}\right)_{+}, \mathfrak{c}\right\}=\left(\kappa_{+}\right)_{+}$by $(2)$ and $\operatorname{card}\left({ }^{*} \mathbb{C} \mid \mathbb{C}\right)^{*}=\left(\kappa_{+}\right)_{+}$ by Corollary 4.2 , since card $\mathbb{C}<\operatorname{dim}\left({ }^{*} \mathbb{C} \mid \mathbb{C}\right)^{*}$.

- If $\kappa=\aleph_{0}$, then $\operatorname{dim}\left({ }^{*} \mathbb{C} \mid \mathbb{C}\right) \leq \mathfrak{c}$ holds trivially since $\operatorname{card}\left({ }^{*} \mathbb{C}\right)=\left(\aleph_{0}\right)_{+}=\mathfrak{c}$. To show that $\operatorname{dim}\left({ }^{*} \mathbb{C} \mid \mathbb{C}\right)=\mathfrak{c}$, we observe that the set $E=\left\{\rho^{r}: r \in \mathbb{R}\right\}$ is a free subset of $* \mathbb{C}$ of cardinality $\mathfrak{c}$. Here $\rho$ is a (fixed) positive infinitesimal in ${ }^{*} \mathbb{C}$ (actually, $\rho \in{ }^{*} \mathbb{R}$ ). The next two formulas, $\operatorname{dim}\left({ }^{*} \mathbb{C} \mid \mathbb{C}\right)^{*}=\operatorname{card}\left({ }^{*} \mathbb{C} \mid \mathbb{C}\right)^{*}=\mathfrak{c}_{+}$, follow by exactly the same arguments as in case $\kappa>\aleph_{0}$.

Example 7.12 (The Space of Nonstandard Polynomials and its Dual) Let $* \mathbb{C}[z]$ denote the vector space over the field $* \mathbb{C}$ consisting of all polynomials in one variable with coefficients in ${ }^{*} \mathbb{C}$. Let $\left({ }^{*}[z]\right)^{*}$ stand for the dual space of $* \mathbb{C}[z]$ (warning again, the two asterisks have different meaning). As in Example 7.5, $\operatorname{dim}\left({ }^{*} \mathbb{C}[z]\right)=\aleph_{0}$, because $\left\{1, z, z^{2}, \ldots\right\}$ is (obviously) a Hamel basis of ${ }^{*} \mathbb{C}[z]$, and $\operatorname{card}\left({ }^{*} \mathbb{C}[z]\right)=\operatorname{dim}\left({ }^{*} \mathbb{C}[z]\right)^{*}=$ $\operatorname{card}\left({ }^{*} \mathbb{C}[z]\right)^{*}=\kappa_{+}$.

Let $\kappa$ be an infinite cardinal and let ${ }^{*} \mathcal{E}(\Omega)$ be the nonstandard extension of $\mathcal{E}(\Omega)=$ $\mathcal{C}^{\infty}(\Omega)$ in a $\kappa_{+}$-saturated ultra-power nonstandard model with set of individuals $\mathbb{R}$ (the same nonstandard framework as in Examples 7.11). We have $\operatorname{card}\left({ }^{*} \mathcal{E}(\Omega)\right)=\kappa_{+}$. Indeed, $\operatorname{card}\left({ }^{*} \mathcal{E}(\Omega)\right) \leq \kappa_{+}$holds, because ${ }^{*} \mathcal{E}(\Omega)=\mathcal{E}(\Omega)^{I} / \mathcal{U}$, where $\operatorname{card}(I)=\kappa$ and $\mathcal{U}$ is a $\kappa_{+}$-good free ultrafilter on $I$ (Chang and Keisler [4] or Lindstrøm [25]). Thus, $\operatorname{card}\left(\mathcal{E}(\Omega)^{I}\right)=\mathfrak{c}^{\kappa}=\kappa_{+}$. On the other hand, $\operatorname{card}\left({ }^{*} \mathcal{E}(\Omega)\right) \geq \kappa_{+}$holds, because ${ }^{*} \mathcal{E}(\Omega)$ is $\kappa_{+}$-saturated. Thus, $\operatorname{card}\left({ }^{*} \mathcal{E}(\Omega)\right)=\kappa_{+}$. With this in mind, we have the following example.

Example 7.13 (The Space ${ }^{*} \mathcal{E}(\Omega) \mid \mathbb{C}$ and its Dual) Let $* \mathcal{E}(\Omega) \mid \mathbb{C}$ denote the vector space of $* \mathcal{E}(\Omega)$ over $\mathbb{C}$ and $\left({ }^{*} \mathcal{E}(\Omega) \mid \mathbb{C}\right)^{*}$ be the algebraic dual of $* \mathcal{E}(\Omega) \mid \mathbb{C}$ (the asterisks in front and after $\mathcal{E}(\Omega)$ have different meaning). We have $\operatorname{card}\left({ }^{*} \mathcal{E}(\Omega)\right)=\operatorname{card}\left({ }^{*} \mathcal{E}(\Omega) \mid \mathbb{C}\right)=$ $\operatorname{dim}\left({ }^{*} \mathcal{E}(\Omega) \mid \mathbb{C}\right)=\kappa_{+}$and $\operatorname{card}\left({ }^{*} \mathcal{E}(\Omega) \mid \mathbb{C}\right)^{*}=\operatorname{dim}\left({ }^{*} \mathcal{E}(\Omega) \mid \mathbb{C}\right)^{*}=\left(\kappa_{+}\right)_{+}$. Indeed:

- If $\kappa>\aleph_{0}$, then $\operatorname{dim}\left({ }^{*} \mathcal{E}(\Omega) \mid \mathbb{C}\right)=\kappa_{+}$by Corollary 3.5, since card $\mathbb{C}<$ $\operatorname{card}\left({ }^{*} \mathcal{E}(\Omega)\right)$. Next, $\operatorname{dim}\left({ }^{*} \mathcal{E}(\Omega) \mid \mathbb{C}\right)^{*}=\max \left\{\left(\kappa_{+}\right)_{+}, \mathfrak{c}\right\}=\left(\kappa_{+}\right)_{+}$by $(2)$ and $\operatorname{card}\left({ }^{*} \mathcal{E}(\Omega) \mid \mathbb{C}\right)^{*}=\left(\kappa_{+}\right)_{+}$by Corollary 4.2 , since card $\mathbb{C}<\operatorname{dim}\left({ }^{*} \mathcal{E}(\Omega) \mid \mathbb{C}\right)^{*}$. 
- If $\kappa=\aleph_{0}$, then $\operatorname{dim}\left({ }^{*} \mathcal{E}(\Omega) \mid \mathbb{C}\right) \leq \mathfrak{c}$ holds trivially since $\operatorname{card}\left({ }^{*} \mathcal{E}(\Omega)\right)=\left(\aleph_{0}\right)_{+}=$ c. To show that $\operatorname{dim}\left({ }^{*} \mathcal{E}(\Omega) \mid \mathbb{C}\right)=\mathfrak{c}$, we observe that the set $E=\left\{e^{\lambda x}: \lambda \in \mathbb{C}\right\}$ is a free subset of ${ }^{*} \mathcal{E}(\Omega)$ of cardinality $\mathfrak{c}$. The next two formulas, $\operatorname{dim}\left({ }^{*} \mathcal{E}(\Omega) \mid \mathbb{C}\right)^{*}=$ $\operatorname{card}\left({ }^{*} \mathcal{E}(\Omega) \mid \mathbb{C}\right)^{*}=\mathfrak{c}_{+}$, follow by exactly the same arguments as in case $\kappa>\aleph_{0}$.

Example 7.14 (The Space $\left.{ }^{*} \mathcal{E}(\Omega)\right|^{*} \mathbb{C}$ and its Dual) Let $\left.{ }^{*} \mathcal{E}(\Omega)\right|^{*} \mathbb{C}$ denote the vector space of ${ }^{*} \mathcal{E}(\Omega)$ over the field ${ }^{*} \mathbb{C}$ and $\left(\left.{ }^{*} \mathcal{E}(\Omega)\right|^{*} \mathbb{C}\right)^{*}$ be its dual space. As in the previous example, we have $\operatorname{card}\left({ }^{*} \mathcal{E}(\Omega)\right)=\operatorname{card}\left(\left.{ }^{*} \mathcal{E}(\Omega)\right|^{*} \mathbb{C}\right)=\operatorname{dim}\left(\left.{ }^{*} \mathcal{E}(\Omega)\right|^{*} \mathbb{C}\right)=\kappa_{+}$and $\operatorname{card}\left(\left.{ }^{*} \mathcal{E}(\Omega)\right|^{*} \mathbb{C}\right)^{*}=\operatorname{dim}\left(\left.{ }^{*} \mathcal{E}(\Omega)\right|^{*} \mathbb{C}\right)^{*}=\left(\kappa_{+}\right)_{+}$. Indeed, $\operatorname{dim}\left(\left.{ }^{*} \mathcal{E}(\Omega)\right|^{*} \mathbb{C}\right) \leq \kappa_{+}$holds trivially, because card $\left({ }^{*} \mathcal{E}(\Omega)\right)=\kappa_{+}$(Example 7.13). To show that $\operatorname{dim}\left(\left.{ }^{*} \mathcal{E}(\Omega)\right|^{*} \mathbb{C}\right)=$ $\kappa_{+}$, we observe that $E=\left\{e^{\lambda x}: \lambda \in{ }^{*} \mathbb{C}\right\}$ is a free subset of $* \mathcal{E}(\Omega)$ of cardinality $\kappa_{+} \cdot \operatorname{Next}, \operatorname{dim}\left(\left.{ }^{*} \mathcal{E}(\Omega)\right|^{*} \mathbb{C}\right)^{*}=\max \left\{\left(\kappa_{+}\right)_{+}, \kappa_{+}\right\}=\left(\kappa_{+}\right)_{+}$by (2). Finally, $\operatorname{card}\left(\left.{ }^{*} \mathcal{E}(\Omega)\right|^{*} \mathbb{C}\right)^{*}=\left(\kappa_{+}\right)_{+}$by Corollary 4.2 , since $\operatorname{card}\left({ }^{*} \mathbb{C}\right)<\operatorname{dim}\left({ }^{*} \mathcal{E}(\Omega) \mid \mathbb{C}\right)^{*}$.

\section{Linear functionals in $\mathcal{D}^{*}(\Omega)$ as generalized functions: the main result}

We supply $\mathcal{D}^{*}(\Omega)$ (Example 7.6) with the structure of a sheaf of differential $\mathbb{C}$-vector spaces (and, more generally, a sheaf of differential modules over $\mathcal{E}(\Omega)=\mathcal{C}^{\infty}(\Omega)$ ). This structure is inherited from $\mathcal{D}(\Omega)$ by duality. Our framework is the infinite-dimensional linear algebra presented in Sections 3-5 applied to particular case $V=\mathcal{D}(\Omega)$ and its dual $V^{*}=\mathcal{D}^{*}(\Omega)$. As before, $\Omega$ stands for a (generic) open subset of $\mathbb{R}^{d}$ (Section 2).

We are trying to convince the reader that $\mathcal{D}^{*}(\Omega)$ deserves to be treated as a space of generalized functions. In what follows the space $\mathcal{L}_{\text {loc }}(\Omega)$ (and more literarily, $S_{\Omega}\left[\mathcal{L}_{\text {loc }}(\Omega)\right]$ explained below) presents the set of classical functions as apposed to the generalized functions in $\mathcal{D}^{*}(\Omega)$. The embedding of Schwartz distributions (Vladimirov [39]) in $\mathcal{D}^{*}(\Omega)$ will be discussed in the next section.

Our approach is a refinement and generalization of distribution theory. That is why, starting from this section, we follow the tradition of distribution theory and use the bracket notation mentioned in (Remark 5.3): We shall write $\langle T, \varphi\rangle$ instead of $T(\varphi)$ for the evaluation of $T \in \mathcal{D}^{*}(\Omega)$ at $\varphi \in \mathcal{D}(\Omega)$.

Definition 8.1 (The Space $\mathcal{D}^{*}(\Omega)$ ) (1) Let $f \in \mathcal{E}(\Omega)$ and $T \in \mathcal{D}^{*}(\Omega)$. We define the product $f T \in \mathcal{D}^{*}(\Omega)$ by $\langle f T, \varphi\rangle=\langle T, f \varphi\rangle$ for all $\varphi \in \mathcal{D}(\Omega)$.

(2) Let $X$ be an open set of $\mathbb{R}^{d}$ such that $X \subseteq \Omega$ and $T \in \mathcal{D}^{*}(\Omega)$. We define the restriction $T\left\lceil X \in \mathcal{D}^{*}(X)\right.$ of $T$ on $X$ by $\langle T \mid X, \varphi\rangle=\langle T, \bar{\varphi}\rangle$ for all $\varphi \in \mathcal{D}(X)$, where $\bar{\varphi}$ is the extension of $\varphi$ from $X$ to $\Omega$ by zero-values on $\Omega \backslash X$. 
(3) Let $T_{n}, T \in \mathcal{D}^{*}(\Omega), n \in \mathbb{N}$. We define the weak (pointwise) convergence $T_{n} \stackrel{*}{\mapsto} T$ in $\mathcal{D}^{*}(\Omega)$ by $\lim _{n \mapsto \infty}\left\langle T_{n}, \varphi\right\rangle=\langle T, \varphi\rangle$ for all $\varphi \in \mathcal{D}(\Omega)$, where $\lim _{n \mapsto \infty}$ is the usual limit in $\mathbb{C}$.

(4) Let $\alpha=\left(\alpha_{1}, \ldots, \alpha_{d}\right) \in \mathbb{N}_{0}^{d}$ be a multi-index and $|\alpha|=\alpha_{1}+\cdots+\alpha_{d}$. Let $\partial^{\alpha}=\frac{\partial^{|\alpha|}}{\partial x_{1}^{\alpha_{1}} \ldots \partial x_{d}^{\alpha_{d}}}$ be the usual partial derivative operator. We define $\partial^{\alpha}$ : $\mathcal{D}^{*}(\Omega) \mapsto \mathcal{D}^{*}(\Omega)$ by $\left\langle\partial^{\alpha} T, \varphi\right\rangle=(-1)^{|\alpha|}\left\langle T, \partial^{\alpha} \varphi\right\rangle$ for all $\varphi \in \mathcal{D}(\Omega)$.

(5) We define the Schwartz embedding $S_{\Omega}: \mathcal{L}_{\text {loc }}(\Omega) \mapsto \mathcal{D}^{*}(\Omega)$ by $S_{\Omega}(f)=T_{f}$, where $\left\langle T_{f}, \varphi\right\rangle=\int_{\Omega} f(x) \varphi(x) d x$ for all $\varphi \in \mathcal{D}(\Omega)$.

(6) Let $X$ and $Y$ be two open sets of $\mathbb{R}^{d}, \theta \in \operatorname{Diff}(X, Y)$ be a diffeomorphism from $X$ to $Y$ and $J_{\theta}: X \rightarrow Y, J_{\theta}=\left|\operatorname{det}\left(\frac{\partial \theta}{\partial x}\right)\right|$, be the corresponding Jacobian determinant. We define the change of variables $\theta_{*}: \mathcal{D}^{*}(X) \rightarrow \mathcal{D}^{*}(Y)$ by the formula $\left\langle\theta_{*}(T)(y), \varphi(y)\right\rangle=\left\langle T(x),(\varphi \circ \theta)(x) J_{\theta}(x)\right\rangle$ for all $\varphi \in \mathcal{D}(Y)$. We sometimes write $T\left(\theta^{-1}\right)$ instead of $\theta_{*}(T)$.

Examples 8.2 (Inflections and Translations) (1) Let $X=Y=\mathbb{R}^{d}$ and $\theta(x)=-x$. We denote the inflection $\theta_{*}(T)$ of $T$ by $\check{T}$, ie $\langle\check{T}, \varphi\rangle=\langle T, \check{\varphi}\rangle$ for all $\varphi \in \mathcal{D}\left(\mathbb{R}^{d}\right)$, where $\check{\varphi}(x)=\varphi(-x)$.

(2) Let (as above) $X=Y=\mathbb{R}^{d}, h \in \mathbb{R}^{d}$ and let $\theta(x)=x+h$. We have $\left\langle\left(\theta_{*} T\right)(y), \varphi(y)\right\rangle=\langle T(x), \varphi(x+h)\rangle$ for all $\varphi \in \mathcal{D}\left(\mathbb{R}^{d}\right)$. It is customary to call the mappings $\tau_{h}: \mathcal{D}^{*}\left(\mathbb{R}^{d}\right) \mapsto \mathcal{D}^{*}\left(\mathbb{R}^{d}\right), \tau_{h} T=\theta_{*}(T)$, translations and often to write $T(y-h)$ instead of $\left(\theta_{*} T\right)(y)$.

Theorem 8.3 (Properties of $\mathcal{D}^{*}(\Omega)$ ) (i) $\mathcal{D}^{*}(\Omega)$ is a differential module over $\mathcal{E}(\Omega)$. Consequently, $\mathcal{D}^{*}(\Omega)$ is a vector space over $\mathbb{C}$ and $S_{\Omega}\left[\mathcal{L}_{\text {loc }}(\Omega)\right]$ is a vector subspace of $\mathcal{D}^{*}(\Omega)$.

(ii) The family $\left\{\mathcal{D}^{*}(\Omega)\right\}_{\Omega \in \mathcal{T}^{d}}$ is a sheaf of differential modules over $\mathcal{E}(\Omega)$ relative to the usual topology $\mathcal{T}^{d}$ on $\mathbb{R}^{d}$ and the restriction $\uparrow$ (Kaneko [21, page 16]). Consequently, the family $\left\{\mathcal{D}^{*}(\Omega)\right\}_{\Omega \in \mathcal{T}^{d}}$ is a sheaf of differential vector spaces over $\mathbb{C}$. (Thus the support, $\operatorname{supp}(T)$, is well-defined for every $T \in \mathcal{D}^{*}(\Omega)$ ).

(iii) The family $\left\{S_{\Omega}\left[\mathcal{L}_{l o c}(\Omega)\right]\right\}_{\Omega \in \mathcal{T}^{d}}$ is a subsheaf of $\left\{\mathcal{D}^{*}(\Omega)\right\}_{\Omega \in \mathcal{T}^{d}}$ of both $\mathcal{E}(\Omega)$ modules and $\mathbb{C}$-vector spaces. Thus $\operatorname{supp}(f)=\operatorname{supp}\left(S_{\Omega}(f)\right)$ for every $f \in$ $\mathcal{L}_{\text {loc }}(\Omega)$.

(iv) The linear partial differential operator $P^{*}(x, \partial)$ : $\mathcal{D}^{*}(\Omega) \mapsto \mathcal{D}^{*}(\Omega), P^{*}(x, \partial)=$ $\sum_{|\alpha| \leq m} c_{\alpha}(x) \partial^{\alpha}$, with $\mathcal{C}^{\infty}$-coefficients $c_{\alpha} \in \mathcal{E}(\Omega)$, is the dual of the operator $P(x, \partial): \mathcal{D}(\Omega) \mapsto \mathcal{D}(\Omega)$, defined by $P(x, \partial) \varphi(x)=\sum_{|\alpha| \leq m}(-1)^{|\alpha|} \partial^{\alpha}\left(c_{\alpha}(x) \varphi(x)\right)$ (Definition 5.2). Consequently, $P^{*}(x, \partial)$ is regular (Definition 5.5)-thus surjective-iff the operator $P(x, \partial)$ is injective. 
Proof (i) follows from the fact that $f \in \mathcal{E}(\Omega)$ and $\varphi \in \mathcal{D}(\Omega)$ implies $f \varphi \in \mathcal{D}(\Omega)$.

(ii) For a short proof we refer to (Oberguggenberger [30, page 10]).

(iii) follows directly from (i).

(iv) We calculate $\left\langle P^{*}(x, \partial) T, \varphi\right\rangle=\sum_{|\alpha| \leq m}\left\langle c_{\alpha} \partial^{\alpha} T, \varphi\right\rangle=\sum_{|\alpha| \leq m}\left\langle\partial^{\alpha} T, c_{\alpha} \varphi\right\rangle=$ $\sum_{|\alpha| \leq m}(-1)^{|\alpha|}\left\langle T, \partial^{\alpha}\left(c_{\alpha} \varphi\right)\right\rangle=\left\langle T, \sum_{|\alpha| \leq m}(-1)^{|\alpha|} \partial^{\alpha}\left(c_{\alpha} \varphi\right)\right\rangle=\langle T, P(x, \partial) \varphi\rangle$ for all $T \in \mathcal{D}^{*}(\Omega)$ and all $\varphi \in \mathcal{D}(\Omega)$. Thus $P^{*}(x, \partial)$ is the dual of $P(x, \partial)$. The rest follows immediately from Theorem 5.4 applied for $V=\mathcal{D}(\Omega)$ and $\mathcal{O}=P(x, \partial)$.

Theorem 8.4 (The Main Result) Let $P^{*}(x, \partial)=\sum_{|\alpha| \leq m} c_{\alpha}(x) \partial^{\alpha}$ be a regular linear partial differential operator with $\mathcal{C}^{\infty}$-coefficients. Then the equation $P^{*}(x, \partial) U=T$ is solvable in $\mathcal{D}^{*}(\Omega)$ in the sense that for any choice of $T$ in $\mathcal{D}^{*}(\Omega)$ this equation has a solution $U$ in $\mathcal{D}^{*}(\Omega)$.

Proof 1. An immediate consequence of Corollary 5.6 applied for $V=\mathcal{D}(\Omega), V^{*}=$ $\mathcal{D}^{*}(\Omega), \mathcal{O}=P(x, \partial)$ and $\mathcal{O}^{*}=P^{*}(x, \partial)$, since the operator $P^{*}(x, \partial)$ is the dual of $P(x, \partial)$ by Theorem 8.3 (iv).

Proof 2. Here is an independent proof based on Theorem 3.6 only. Let $\operatorname{ran}(P(x, \partial)) \subseteq$ $\mathcal{D}(\Omega)$ denote the range of $P(x, \partial)$. We define the linear functional $\Phi: \operatorname{ran}(P(x, \partial)) \mapsto \mathbb{C}$ by $\langle\Phi, P(x, \partial) \varphi\rangle=\langle T, \varphi\rangle$ for all $\varphi \in \mathcal{D}(\Omega)$. The mapping $\Phi$ is well defined, since $P(x, \partial)$ is injective by assumption, and $\Phi \in(\operatorname{ran}(P(x, \partial)))^{*}$, because $T$ is linear. Let $\mathcal{B}_{1}$ be a (Hamel) basis of $\operatorname{ran}(P(x, \partial))$. Then $\mathcal{B}_{1}$ can be extended to a basis $\mathcal{B}$ of $\mathcal{D}^{*}(\Omega)$ by Theorem 3.6. We define $U \in \mathcal{D}^{*}(\Omega)$ by $U(\psi)=\Phi(\psi)$ for all $\psi \in \mathcal{B}_{1}$ and $U(\psi)=0$ (or anyhow) for $\psi \in \mathcal{B} \backslash \mathcal{B}_{1}$. It is clear that $U$ is an extension of $\Phi$ from $\operatorname{ran}(P(x, \partial)$ ) to $\mathcal{D}^{*}(\Omega)$. Thus $\left\langle P^{*}(x, \partial) U, \varphi\right\rangle=\langle U, P(x, \partial) \varphi\rangle=\langle\Phi, P(x, \partial) \varphi\rangle=\langle T, \varphi\rangle$, for all $\varphi \in \mathcal{D}(\Omega)$, as required.

\section{Schwartz distributions within $\mathcal{D}^{*}(\Omega)$ : sequential approach to distribution theory}

We characterize the space of Schwartz distributions $\mathcal{D}^{\prime}(\Omega)$ as a particular subspace of $\mathcal{D}^{*}(\Omega)$ without involving the usual strong topology on the space of test-functions $\mathcal{D}(\Omega)$ (Vladimirov [39]). We discuss the similarities and differences between $\mathcal{D}^{\prime}(\Omega)$ and $\mathcal{D}^{*}(\Omega)$ and give a short outline of a sequential approach to distribution theory based on our characterization. 
Theorem 9.1 (Connection to Schwartz Distributions)

(i) The space $\mathcal{D}^{\prime}(\Omega)$ of Schwartz distributions on $\Omega$ (Vladimirov [39]) coincides with the weak sequential completion of $\mathcal{L}_{\text {loc }}(\Omega)$, ie

$$
\mathcal{D}^{\prime}(\Omega)=\left\{T \in \mathcal{D}^{*}(\Omega):\left(\exists\left(T_{n}\right) \in\left(S_{\Omega}\left[\mathcal{L}_{l o c}(\Omega)\right]\right)^{\mathbb{N}}\right)\left(T_{n} \stackrel{*}{\mapsto} T\right)\right\}
$$

where $S_{\Omega}\left[\mathcal{L}_{\text {loc }}(\Omega)\right]$ is the image of $\mathcal{L}_{\text {loc }}(\Omega)$ under $S_{\Omega}$ (Definition 8.1) and $\left(S_{\Omega}\left[\mathcal{L}_{\text {loc }}(\Omega)\right]\right)^{\mathbb{N}}$ denote the space of all sequences in $S_{\Omega}\left[\mathcal{L}_{\text {loc }}(\Omega)\right]$. (Compare with Remark 9.2 below.)

(ii) $\mathcal{D}^{\prime}(\Omega)$ is a differential $\mathcal{E}(\Omega)$-submodule of $\mathcal{D}^{*}(\Omega)$. Consequently, $\mathcal{D}^{\prime}(\Omega)$ is a differential $\mathbb{C}$-vector subspace of $\mathcal{D}^{*}(\Omega)$.

(iii) The family $\left\{\mathcal{D}^{\prime}(\Omega)\right\}_{\Omega \in \mathcal{T}^{d}}$ is a subsheaf of $\left\{\mathcal{D}^{*}(\Omega)\right\}_{\Omega \in \mathcal{T}^{d}}$ of differential $\mathcal{E}(\Omega)$ modules (and $\mathbb{C}$-vector spaces) (Definition 8.1), where $\mathcal{T}^{d}$ stands for the usual topology on $\mathbb{R}^{d}$. Consequently, the support $\operatorname{supp}(T)$ in $\mathcal{D}^{*}(\Omega)$ coincides with the usual support of $T$ in distribution theory $[39, \S 1.5$, page 16], for every distribution $T$.

(iv) The inclusion $\mathcal{D}^{\prime}(\Omega) \subset \mathcal{D}^{*}(\Omega)$ is invariant under diffeomorphisms $\theta \in \operatorname{Diff}(X, Y)$.

Proof (i) follows from the fact that the space of Schwartz distributions is sequentially complete under the weak convergence and every distribution can be regularized within $\mathcal{D}(\Omega)$. For a detailed proof we refer the reader to $[39, \S 1.4$, page 14 and $\S 4.6$, pages $80-81$ ].

(ii) The product $f T$ in $\mathcal{E}(\Omega) \times \mathcal{D}^{*}(\Omega)$ (Definition 8.1) coincides with the product $f T$ in $\mathcal{E}(\Omega) \times \mathcal{D}^{\prime}(\Omega)$ in the case when $T$ is a distribution.

(iii) The definition of restriction $T\left\lceil X\right.$ in $\mathcal{D}^{*}(\Omega)$ (Definition 8.1) coincides with the definition of restriction $T \nmid X$ in the Schwartz theory of distributions [39, §1.3, page 12] in the case when $T$ is a distribution.

(iv) The definition of change of variables $Q_{*}(T)$ in $\mathcal{D}^{*}(\Omega)$ (Definition 8.1) coincides with the definition of change of variables $Q_{*}(T)$ in the Schwartz theory of distributions (Hörmander $[15, \S 6.1]$ ) in the case when $T$ is a distribution.

Remark 9.2 (Sequential Approach to Distribution Theory) (1) The formula (4) can be written in the form:

$$
\begin{aligned}
\mathcal{D}^{\prime}(\Omega)=\left\{T \in \mathcal{D}^{*}(\Omega):\left(\exists\left(f_{n}\right) \in\left(\mathcal{L}_{l o c}(\Omega)\right)^{\mathbb{N}}\right)(\forall \varphi \in \mathcal{D}(\Omega))\right. \\
\left.\lim _{n \mapsto \infty} \int_{\Omega} f_{n}(x) \varphi(x) d x=\langle T, \varphi\rangle\right\}
\end{aligned}
$$

where $\left(\mathcal{L}_{l o c}(\Omega)\right)^{\mathbb{N}}$ denotes the space of all sequences in $\mathcal{L}_{\text {loc }}(\Omega)$ (Section 2) and the "lim" stands for the usual limit in $\mathbb{C}$. 
(2) The space $\mathcal{L}_{\text {loc }}(\Omega)$ in (5) can be replaced by $\mathcal{E}(\Omega)=\mathcal{C}^{\infty}(\Omega)$ and even by $\mathcal{D}(\Omega)=$ $\mathcal{C}_{0}^{\infty}(\Omega)[39, \S 4.6$, page 79]. This gives rise to the following sequential definition of Schwartz distributions $\mathcal{D}^{\prime}(\Omega)=F\left(\mathcal{E}(\Omega)^{\mathbb{N}}\right) / \sim$. Here $F\left(\mathcal{E}(\Omega)^{\mathbb{N}}\right)$ denotes the set of all fundamental sequences $\left(f_{n}\right)$ in $\mathcal{E}(\Omega)$, ie the sequences with the property that for every (fixed) test function $\varphi \in \mathcal{D}(\Omega)$ the sequence $\left(\int_{\Omega} f_{n}(x) \varphi(x) d x\right)$ is fundamental (convergent) in $\mathbb{C}$. Also, $\sim$ stands for the equivalence relation on $F\left(\mathcal{E}(\Omega)^{\mathbb{N}}\right)$ defined by $\left(f_{n}\right) \sim\left(g_{n}\right)$ if $\lim _{n \mapsto \infty} \int_{\Omega}\left(f_{n}(x)-g_{n}(x)\right) \varphi(x) d x=0$ for all $\varphi \in \mathcal{D}(\Omega)$. For a similar sequential approach, we refer to Lighthill [24].

Theorem 9.3 $\mathcal{D}^{*}(\Omega) \backslash \mathcal{D}^{\prime}(\Omega) \neq \varnothing$.

Proof 1. $\mathcal{D}^{\prime}(\Omega)$ is a vector subspace of $\mathcal{D}^{*}(\Omega)$, and $\operatorname{dim}\left(\mathcal{D}^{\prime}(\Omega)\right)=\mathfrak{c}$, and $\operatorname{dim}\left(\mathcal{D}^{*}(\Omega)\right)=$ $\mathfrak{c}_{+}$(Example 7.6). Thus $\mathcal{D}^{\prime}(\Omega)$ is a proper subspace of $\mathcal{D}^{*}(\Omega)$ by Lemma 3.9.

Proof 2. Let $\left\{\varphi_{0}, \varphi_{1}, \varphi_{2}, \ldots\right\}$ be a free set of $\mathcal{D}(\Omega)$ such that $\varphi_{n} \mapsto \varphi_{0}$ as $n \mapsto \infty$, in the strong topology of $\mathcal{D}(\Omega)$ (Vladimirov [39], §1.2, page 7). Since $\operatorname{dim}(\mathcal{D}(\Omega))=\mathfrak{c}$, there exists a (Hamel) basis $\left\{\varphi_{r}: r \in \mathbb{R}\right\}$ of $\mathcal{D}(\Omega)$ which extends the sequence $\left\{\varphi_{0}, \varphi_{1}, \varphi_{2}, \ldots\right\}$ by Theorem 3.6. Define $T \in \mathcal{D}^{*}(\Omega)$ by $T\left(\varphi_{0}\right)=1$ and $T\left(\varphi_{r}\right)=0$, $r \in \mathbb{R}, r \neq 0$. Thus $T \in \mathcal{D}^{*}(\Omega) \backslash \mathcal{D}^{\prime}(\Omega)$ (as required) by Theorem 9.1(i), since $\lim _{n \mapsto \infty} T\left(\varphi_{n}\right)=0 \neq 1=T\left(\varphi_{0}\right)$.

Proof 3. For the existence of discontinuous linear functionals (based on a Borel theorem), we refer to Oberguggenberger [30, Example 10, page 11].

We borrow the next definition and the following lemma and theorem from [30, page 15].

Definition 9.4 (Convolution) Let $S \in \mathcal{D}^{*}\left(\mathbb{R}^{d}\right)$ or $S \in \mathcal{E}^{*}\left(\mathbb{R}^{d}\right)$. Let $T \in \mathcal{D}^{\prime}\left(\mathbb{R}^{d}\right)$. We define the convolution $S \star T \in \mathcal{D}^{*}\left(\mathbb{R}^{d}\right)$ by

$$
\langle S \star T, \varphi\rangle=\langle S, \check{T} \star \varphi\rangle
$$

for all $\varphi \in \mathcal{D}\left(\mathbb{R}^{d}\right)$, where $\check{T}$ is the inflection of $T$ (Example 8.2) and $\check{T} * \varphi$ is the usual convolution in the sense of distribution theory (Vladimirov [39, Chapter 4]).

Lemma 9.5 $\partial^{\alpha}(S \star T)=\left(\partial^{\alpha} S\right) \star T=S \star\left(\partial^{\alpha} T\right)$ for all multi-indexes $\alpha \in \mathbb{N}_{0}^{d}$.

Proof Let $\varphi \in \mathcal{D}\left(\mathbb{R}^{d}\right)$. We calculate $\left\langle\partial^{\alpha}(S \star T), \varphi\right\rangle=(-1)^{|\alpha|}\left\langle S, \check{T} \star \partial^{\alpha} \varphi\right\rangle=$ $(-1)^{|\alpha|}\left\langle S, \partial^{\alpha}(\check{T} \star \varphi)\right\rangle$. On the other hand, $(-1)^{|\alpha|}\left\langle S, \partial^{\alpha}(\check{T} \star \varphi)\right\rangle=\left\langle\left(\partial^{\alpha} S\right) \star T, \varphi\right\rangle$. Thus $\partial^{\alpha}(S \star T)=\left(\partial^{\alpha} S\right) \star T$ as required. Also, $(-1)^{|\alpha|}\left\langle S, \partial^{\alpha}(\check{T} \star \varphi)\right\rangle=(-1)^{|\alpha|}\left\langle S,\left(\partial^{\alpha} \breve{T}\right) \star \varphi\right\rangle=$ $\left.\left.\left\langle S,\left(\partial^{\alpha} T\right) \star \varphi\right)\right\rangle=\left\langle S \star \partial^{\alpha} T, \varphi\right)\right\rangle$. Thus $\partial^{\alpha}(S \star T)=S \star \partial^{\alpha} T$ as required. 
Theorem 9.6 (Fundamental Solutions) Let $c_{\alpha} \in \mathbb{C}, \alpha \in \mathbb{N}_{0}^{d},|\alpha| \leq m$ and $P^{*}(\partial)$ : $\mathcal{D}^{*}\left(\mathbb{R}^{d}\right) \mapsto \mathcal{D}^{*}\left(\mathbb{R}^{d}\right), P^{*}(\partial)=\sum_{|\alpha| \leq m} c_{\alpha} \partial^{\alpha}$, be the corresponding linear partial differential operator with constant coefficients. Then:

(i) The operator $P^{*}(\partial)$ has a fundamental solution $F \in \mathcal{D}^{*}(\Omega)$, a solution of the equation $P^{*}(\partial) F=\delta$ in $\mathcal{D}^{*}(\Omega)$.

(ii) Let $F \in \mathcal{D}^{*}(\Omega)$ be a fundamental solution of $P^{*}(\partial)$ and $T \in \mathcal{E}^{\prime}(\Omega)$. Then $U=F \star T$ is a solution of $P^{*}(\partial) U=T$ in $\mathcal{D}^{*}(\Omega)$.

(iii) Let $F \in \mathcal{D}^{\prime}(\Omega)$ be a fundamental solution of $P^{*}(\partial)$ and $T \in \mathcal{D}^{*}(\Omega)$ (or even $T \in \mathcal{E}^{*}(\Omega)$ ). Then $U=F \star T$ is a solution of $P^{*}(\partial) U=T$ in $\mathcal{D}^{*}(\Omega)$.

Proof (i) follows directly from Theorem 8.4, because $P^{*}(\partial)$ is a regular operator (Section 11) and $\mathcal{D}^{\prime}(\Omega) \subset \mathcal{D}^{*}(\Omega)$ (thus $\delta \in \mathcal{D}^{*}(\Omega)$ ). In both (i) and (ii) we have $\left.P^{*}(\partial) U=P^{*}(\partial)(F \star T)=\left(P^{*}(\partial) F\right) \star T\right)=\delta \star T=T$. The last equality, $\delta \star T=T$, is derived in (ii) and (iii) somewhat differently:

(ii) $\langle T \star \delta, \varphi\rangle=\langle\delta, \breve{T} \star \varphi\rangle=(\breve{T} \star \varphi)(0)=\langle T, \varphi\rangle$, for all $\varphi \in \mathcal{D}\left(\mathbb{R}^{d}\right)$.

(iii) $\langle T \star \delta, \varphi\rangle=\langle T, \check{\delta} \star \varphi\rangle=\langle T, \delta \star \varphi\rangle=\langle T, \varphi\rangle$, for all $\varphi \in \mathcal{D}\left(\mathbb{R}^{d}\right.$ ) (or even for all $\left.\varphi \in \mathcal{E}\left(\mathbb{R}^{d}\right)\right)$.

Remark 9.7 (Comparison: $\mathcal{D}^{\prime}(\Omega)$ versus $\mathcal{D}^{*}(\Omega)$ ) There are obvious similarities between the properties of $\mathcal{D}^{*}(\Omega)$ (Theorem 8.3) and the space of Schwartz distributions $\mathcal{D}^{\prime}(\Omega)$ (Vladimirov [39]; see also Remark 9.2 in this paper). However, there are also essential differences; here are some of them:

(1) The discontinuous (relative to the strong topology on $\mathcal{D}(\Omega)$ ) linear functionals $T \in$ $\mathcal{D}^{*}(\Omega) \backslash \mathcal{D}^{\prime}(\Omega)$ cannot be approached by a sequence of classical functions in the sense that there is no sequence $\left(f_{n}\right)$ in $\mathcal{L}_{l o c}(\Omega)$ such that $f_{n} \stackrel{*}{\mapsto} T$ (Definition 8.1). In a sense the spaces $\mathcal{L}_{\text {loc }}(\Omega)$ (more precisely, $S\left[\mathcal{L}_{\text {loc }}(\Omega)\right]$ ), $\mathcal{D}^{\prime}(\Omega)$ and $\mathcal{D}^{*}(\Omega)$ resemble $\mathbb{Q}, \mathbb{R}$ and $\mathbb{C}$, respectively: every real number is the limit of some (fundamental) sequence in $\mathbb{Q}$, but the complex numbers of the for form $a+i b, b \neq$ 0 , can not be approximated by sequences in $\mathbb{Q}$ (we are unaware of subspace of $\mathcal{D}^{*}(\Omega)$ which plays the role of $\mathbb{Q}(i)$ in the above analogy).

(2) The structural theorem for $\mathcal{D}^{\prime}(\Omega)\left[39, \S 2.4\right.$, page 41] fails in $\mathcal{D}^{*}(\Omega)$. Recall that the structural theorem states that for every Schwartz distribution $T \in \mathcal{D}^{\prime}(\Omega)$ and for every open set $X$ of $\mathbb{R}^{d}$, such that $X \subset \subset \Omega$, there exist a classical function $f \in \mathcal{L}^{\infty}(X)$ and a multi-index $\alpha \in \mathbb{N}_{0}^{d}$ such that $T=\partial^{\alpha} f$ in $\mathcal{D}^{\prime}(X)$. This theorem fails in $\mathcal{D}^{*}(\Omega)$.

(3) The convolution (Definition 9.4) fails to regularize $T \in \mathcal{D}^{*}\left(\mathbb{R}^{d}\right) \backslash \mathcal{D}^{\prime}\left(\mathbb{R}^{d}\right)$ in the sense that $T \star \varphi \in \mathcal{E}\left(\mathbb{R}^{d}\right)$ does not necessarily hold for all $\varphi \in \mathcal{D}\left(\mathbb{R}^{d}\right)$.

(4) The direct (tensor) product in $\mathcal{D}^{\prime}(\Omega)\left[39, \S 3\right.$, page 46] also fails in $\mathcal{D}^{*}(\Omega)$. 
(5) From the above list it seems that the space $\mathcal{D}^{\prime}(\Omega)$ is superior over $\mathcal{D}^{*}(\Omega)$ at least from the point of view of partial differential operator theory. As we shall see in the next sections however, the regular operators $P^{*}(x, \partial)$ are (always) surjective on $\mathcal{D}^{*}(\Omega)$, but their restrictions on $\mathcal{D}^{\prime}(\Omega)$ are often not. That means that the partial differential equations of the form $P^{*}(x, \partial) U=T$ are always solvable for $U$ in $\mathcal{D}^{*}(\Omega)$, but not necessarily solvable in $\mathcal{D}^{\prime}(\Omega)$. The latter property of $\mathcal{D}^{*}(\Omega)$ is the main reason why we believe that the space $\mathcal{D}^{*}(\Omega)$-rather than $\mathcal{D}^{\prime}(\Omega)$ - should be considered as the natural framework of partial differential equations, especially the linear ones with smooth coefficients.

\section{Three invariant subspaces}

In order to prepare better for the discussion in the next section, we select three important subspaces of $\mathcal{D}^{*}(\Omega)$ which are invariant under the linear partial differential operators with $\mathcal{C}^{\infty}$-coefficients.

Lemma 10.1 (Three Invariant Spaces) Let $P^{*}(x, \partial): \mathcal{D}^{*}(\Omega) \mapsto \mathcal{D}^{*}(\Omega), P^{*}(x, \partial)=$ $\sum_{|\alpha| \leq m} c_{\alpha}(x) \partial^{\alpha}$, be a linear partial differential operator (regular or not) with $\mathcal{C}^{\infty}$ coefficients $c_{\alpha} \in \mathcal{E}(\Omega)$. The subspaces of the test-functions $\mathcal{D}(\Omega)=\mathcal{C}_{0}^{\infty}(\Omega)$, the $\mathcal{C}^{\infty}$-functions $\mathcal{E}(\Omega)=\mathcal{C}^{\infty}(\Omega)$ and the $S$ chwartz distributions $\mathcal{D}^{\prime}(\Omega)$ are all invariant under $P^{*}(x, \partial)$.

Proof An immediate consequence from fact that the multiplication by a smooth function on $\mathcal{D}^{*}(\Omega)$ (Definition 8.1(1)) and differentiation on $\mathcal{D}^{*}(\Omega)$ (Definition 8.1(4)) both coincide on $\mathcal{E}(\Omega)$ with the usual multiplication and usual differentiation; and on $\mathcal{D}^{\prime}(\Omega)$ - with the operations with the same names in the sense of Schwartz theory of distributions (Vladimirov [39]).

\section{Examples of regular operators on $\mathcal{D}^{*}(\Omega)$ : solvable partial differential equations}

Following Todorov [37], Oberguggenberger and Todorov [32] and Oberguggenberger [30], we present several examples of regular operators (Definition 5.5) of the form $P^{*}(x, \partial): \mathcal{D}^{*}(\Omega) \mapsto \mathcal{D}^{*}(\Omega), P^{*}(x, \partial)=\sum_{|\alpha| \leq m} c_{\alpha}(x) \partial^{\alpha}$, with $\mathcal{C}^{\infty}$-coefficients $c_{\alpha} \in \mathcal{E}(\Omega)$. Recall that $P^{*}(x, \partial)$ is called regular if its co-dual $P(x, \partial): \mathcal{D}(\Omega) \mapsto \mathcal{D}(\Omega)$, 
$P(x, \partial) \varphi(x)=\sum_{|\alpha| \leq m}(-1)^{|\alpha|} \partial^{\alpha}\left(c_{\alpha}(x) \varphi(x)\right)$, is injective on $\mathcal{D}(\Omega)$ (Theorem 8.3(iv)). Recall as well that the regular operators are exactly the operators for which our main result (Theorem 8.4) holds, ie these are surjective operators on $\mathcal{D}^{*}(\Omega)$.

The restrictions $P^{*}(x, \partial)\left\lceil\mathcal{D}(\Omega), P^{*}(x, \partial) \uparrow \mathcal{E}(\Omega)\right.$ and $P^{*}(x, \partial) \uparrow \mathcal{D}^{\prime}(\Omega)$ of the regular operators $P^{*}(x, \partial)$ in our examples below are relatively well-studied in the classical theory of linear partial differential operators (Hörmander [15]-[17]). With few exceptions, these restrictions are non-surjective. Thus we arrived at the main point of our approach - to extend non-surjective operators from $\mathcal{D}^{\prime}(\Omega)$ to surjective operators on $\mathcal{D}^{*}(\Omega)$ and thus to guarantee the existence of solutions for the corresponding partial differential equations in the framework of $\mathcal{D}^{*}(\Omega)$.

Example 11.1 (Constant Coefficients) The linear partial differential operators with constant coefficients, $P^{*}(\partial): \mathcal{D}^{*}(\Omega) \mapsto \mathcal{D}^{*}(\Omega)$ where $P^{*}(\partial)=\sum_{|\alpha| \leq m} c_{\alpha} \partial^{\alpha}$, are regular. To show that, we have to show that its co-dual (transposed) operator $P(\partial)$ : $\mathcal{D}(\Omega) \mapsto \mathcal{D}(\Omega), P(\partial)=\sum_{|\alpha| \leq m} c_{\alpha}(-1)^{|\alpha|} \partial^{\alpha}$, is injective. Indeed, the Fourier transform applied to $P(\partial) \varphi=0$ leads us to $\left(\sum_{|\alpha| \leq m} c_{\alpha}(i z)^{\alpha}\right) \mathcal{F}(\varphi)(z)=0$. Now, both $\sum_{|\alpha| \leq m} c_{\alpha}(i z)^{\alpha}$ and $\mathcal{F}(\varphi)$ are entire functions and thus the function $\sum_{|\alpha| \leq m} c_{\alpha}(i z)^{\alpha}$ can be cancelled, because the ring of entire functions forms an integral domain (a ring without zero-divisors). The result is $\mathcal{F}(\varphi)(z)=0$, which implies $\varphi=0$ (as required). Consequently, $P^{*}(\partial)$ is surjective on $\mathcal{D}^{*}(\Omega)$, ie the equation $P^{*}(\partial) U=T$ has a solution for $U$ in $\mathcal{D}^{*}(\Omega)$ for every choice of $T$ also in $\mathcal{D}^{*}(\Omega)$. Recall that the restrictions $P^{*}(\partial)\left\lceil\mathcal{D}(\Omega), P^{*}(\partial)\left\lceil\mathcal{E}(\Omega)\right.\right.$ and $P^{*}(\partial)\left\lceil\mathcal{D}^{\prime}(\Omega)\right.$ are not necessarily surjective. In more detail the situation is as follows:

- In the particular case $\Omega=\mathbb{R}^{d}$ the operators $P^{*}(\partial)\left\lceil\mathcal{D}\left(\mathbb{R}^{d}\right)\right.$ is neither surjective nor injective (think of harmonic functions), but the operator $P^{*}(\partial) \uparrow \mathcal{E}\left(\mathbb{R}^{d}\right)$ is surjective, since every convex open subset of $\mathbb{R}^{d}$ is $P^{*}$-convex for supports (Hörmander [14, Theorem 10.6.2]). The operator $P^{*}(\partial)\left\lceil\mathcal{D}^{\prime}\left(\mathbb{R}^{d}\right)\right.$ is also surjective: this is the famous existence theorem of Malgrange [28] and Ehrenpreis [9].

- If $\Omega \neq \mathbb{R}^{d}$ however, the operators $P^{*}(\partial)\left\lceil\mathcal{D}^{\prime}(\Omega)\right.$ might be also non-surjective. In particular, if $P^{*}(\partial)\left\lceil\mathcal{D}^{\prime}(\Omega)\right.$ is non-elliptic, then there exists an open set $\Omega$ of $\mathbb{R}^{d}$ (which is not $P^{*}$-convex for supports) and a smooth function $f \in \mathcal{E}(\Omega)$ such that the equation $P^{*}(\partial) U=f$ has no distributional solution for $U$ [14, Theorem 10.6.6, Corollary 10.6.8, Theorem 10.8.2]. By our result (Theorem 8.4) however, $P^{*}(\partial) U=f$ still does have a solution $U \in \mathcal{D}^{*}(\Omega) \backslash \mathcal{D}^{\prime}(\Omega)$. In addition, we observe that $P^{*}(\partial)$ is certainly non-hypoelliptic on $\mathcal{D}^{*}(\Omega)$ even if its restriction $P^{*}(\partial)\left\lceil\mathcal{D}^{\prime}(\Omega)\right.$ is hypoelliptic, because the solution $U$ is non-smooth.

- For a recent discussion on hypoellipticity we refer to Street [36]. 
Example 11.2 (Elliptic Operators) All second order elliptic operators with $\mathcal{C}^{\infty}$ coefficients $P^{*}(x, \partial): \mathcal{D}^{*}(\Omega) \mapsto \mathcal{D}^{*}(\Omega)$ are regular. This follows from the fact that every test-function $\varphi \in \mathcal{D}(\Omega)$ vanishes on an open set $\Omega$ of $\mathbb{R}^{d}$ and thus $P(x, \partial) \varphi=0$ implies $\varphi=0$ by the Cauchy uniqueness property (uniqueness continuation principle), since $P(x, \partial)$ is also a second order elliptic operator with $\mathcal{C}^{\infty}$-coefficients. Consequently, $P^{*}(x, \partial)$ is surjective on $\mathcal{D}^{*}(\Omega)$, ie the equation $P^{*}(x, \partial) U=T$ has a solution for $U$ in $\mathcal{D}^{*}(\Omega)$ for every choice of $T$ also in $\mathcal{D}^{*}(\Omega)$. We should mention that the restrictions $P^{*}(x, \partial)\left\lceil\mathcal{D}(\Omega), P^{*}(x, \partial)\left\lceil\mathcal{E}(\Omega)\right.\right.$ and $P^{*}(x, \partial)\left\lceil\mathcal{D}^{\prime}(\Omega)\right.$ are not necessarily surjective. For more details we refer to (Hörmander [15, 8.5-8.6], [16, 17.2], and [17, 28.1-28.4]).

Example 11.3 (Analytic Coefficients) All elliptic operators with analytic coefficients $P^{*}(x, \partial): \mathcal{D}^{*}(\Omega) \mapsto \mathcal{D}^{*}(\Omega)$ are regular since their co-dual (transposed) operators $P(x, \partial)$ : $\mathcal{D}(\Omega) \mapsto \mathcal{D}(\Omega)$ are also elliptic with analytic coefficients. More generally, all linear partial differential operators $P^{*}(x, \partial): \mathcal{D}^{*}(\Omega) \mapsto \mathcal{D}^{*}(\Omega)$ with analytic coefficients of constant strength are regular (see the discussion before Theorem 13.5.2 in Hörmander [14, page 196]). Consequently, $P^{*}(x, \partial)$ is surjective on $\mathcal{D}^{*}(\Omega)$, ie the equation $P^{*}(x, \partial) U=$ $T$ has a solution for $U$ in $\mathcal{D}^{*}(\Omega)$ for every choice of $T$ also in $\mathcal{D}^{*}(\Omega)$. We should note that the restrictions $P^{*}(x, \partial)\left\lceil\mathcal{D}(\Omega), P^{*}(x, \partial)\left\lceil\mathcal{E}(\Omega)\right.\right.$ and $P^{*}(x, \partial)\left\lceil\mathcal{D}^{\prime}(\Omega)\right.$ are not necessarily surjective. For the local solvability of equations of the form $P^{*}(x, \partial) U=T$ (which is quite a different phenomenon) and for a more comprehensive discussion on the solvability in general, we refer to Treves [38].

Example 11.4 (Hans Lewy Operator) The Hans Lewy operator

$$
L^{*}(x, \partial): \mathcal{D}^{*}\left(\mathbb{R}^{3}\right) \mapsto \mathcal{D}^{*}\left(\mathbb{R}^{3}\right), \quad L^{*}(x, \partial)=\frac{\partial}{\partial x_{1}}+i \frac{\partial}{\partial x_{2}}-2 i\left(x_{1}+i x_{2}\right) \frac{\partial}{\partial x_{3}}
$$

is regular by the following reasoning (also due to Lewy [23]). First, we verify that $L(x, \partial)=-L^{*}(x, \partial)$. Next, we observe that the mapping $\left(x_{1}, x_{2}, x_{3}\right) \mapsto\left(x_{1}+i x_{2}, x_{3}+\right.$ $\left.i\left(x_{1}^{2}+x_{2}^{2}\right)\right)$ is an embedding of $\mathbb{R}^{3}$ into $\mathbb{C}^{2}$, which converts $\mathbb{R}^{3}$ into the boundary of the domain $D=\left\{\operatorname{Im}\left(z_{2}\right)>\left|z_{1}\right|^{2}\right\}$. Thus every solution $\varphi \in \mathcal{D}\left(\mathbb{R}^{3}\right)$ of $L(x, \partial) \varphi=0$ can be extended holomorphically into $D$. Thus $\varphi=0$ by the unique continuation for holomorphic functions. Consequently, $L^{*}(x, \partial)$ is surjective on $\mathcal{D}^{*}\left(\mathbb{R}^{3}\right)$, ie the equation $L^{*}(x, \partial) U=T$ has a solution for $U$ in $\mathcal{D}^{*}\left(\mathbb{R}^{3}\right)$ for every choice of $T$ also in $\mathcal{D}^{*}\left(\mathbb{R}^{3}\right)$. By contrast, recall that the Lewy operator $L^{*}(x, \partial)\left\lceil\mathcal{D}\left(\mathbb{R}^{3}\right), L^{*}(x, \partial)\left\lceil\mathcal{E}\left(\mathbb{R}^{3}\right)\right.\right.$ and $L^{*}(x, \partial)\left\lceil\mathcal{D}^{\prime}\left(\mathbb{R}^{3}\right)\right.$ are non-surjective and the equation $L^{*}(x, \partial) U=T$ might fail to have solutions $U$ in $\mathcal{D}^{\prime}\left(\mathbb{R}^{3}\right)$ even for some test-functions $T \in \mathcal{D}\left(\mathbb{R}^{3}\right)$ ([23]).

With different arguments (due to Oberguggenberger [30, Remark 20(c), page 18]) we show that the Lewy operator $L^{*}(x, \partial): \mathcal{D}^{*}(\Omega) \mapsto \mathcal{D}^{*}(\Omega)$ is regular for an arbitrary 
open set $\Omega$ of $\mathbb{R}^{3}$ (not only for $\Omega=\mathbb{R}^{3}$ ): we apply the Fourier transform $\mathcal{F}_{3}$ relative to the variable $x_{3}$ - to $L(x, \partial) \varphi\left(x_{1}, x_{2}, x_{3}\right)=0$. The result is $\left(-\frac{\partial}{\partial x_{1}}-i \frac{\partial}{\partial x_{2}}+\right.$ $\left.2\left(x_{1}+i x_{2}\right) z_{3}\right) \psi\left(x_{1}, x_{2}, z_{3}\right)=0$, where $\psi\left(x_{1}, x_{2}, z_{3}\right)=\mathcal{F}_{3}(\varphi)$ is an entire function in $z_{3}$ (for any fixed $\left(x_{1}, x_{2}\right) \in \mathbb{R}^{2}$ such that $\left(x_{1}, x_{2}, x_{3}\right) \in \Omega$ for some $x_{3} \in \mathbb{R}$ ). Next, we let $x_{1}+i x_{2}=z_{2}$ and the result is $\left(\frac{\partial}{\partial \bar{z}_{2}}+2 z_{2} z_{3}\right) \psi\left(z_{2}, z_{3}\right)=0$. The latter implies that the function $z_{2} \mapsto \psi\left(z_{2}, 0\right)$ is analytic and thus (having a compact support) vanishes identically in the variable $z_{2}$. On the other hand, by differentiating $\left(\frac{\partial}{\partial \bar{z}_{2}}+2 z_{2} z_{3}\right) \psi\left(z_{2}, z_{3}\right)=0$ consecutively and letting $z_{3}=0$, we get $\frac{\partial^{k}}{\partial_{z_{3}}} \psi\left(z_{2}, 0\right)=0$ for all $z_{2}$ and all $k \in \mathbb{N}_{0}$. Thus, $\psi=0$ (since, again, $z_{3} \mapsto \psi\left(z_{2}, z_{3}\right)$ is analytic). The latter implies $\varphi=0$ proving that $L^{*}(x, \partial)$ is regular. Consequently, $L^{*}(x, \partial)$ is surjective on $\mathcal{D}^{*}(\Omega)$, ie the equation $L^{*}(x, \partial) U=T$ has a solution for $U$ in $\mathcal{D}^{*}(\Omega)$ for every choice of $T$ also in $\mathcal{D}^{*}(\Omega)$ and any open $\Omega$. By contrast, $L^{*}(x, \partial)\left\lceil\mathcal{D}^{\prime}(\Omega)\right.$ is not necessarily surjective (see above).

Examples 11.5 (Non-Regular Operators) Here are several examples of linear partial differential operators with smooth coefficients which are non-regular:

(1) Let $\psi \in \mathcal{D}(\Omega)$. Then the operator $P^{*}(x, \partial)$ : $\mathcal{D}^{*}(\Omega) \mapsto \mathcal{D}^{*}(\Omega), P^{*}(x, \partial)=\psi \partial^{\alpha}$ is non-regular, because $P(x, \partial) \varphi=(-1)^{|\alpha|} \partial^{\alpha}(\psi \varphi)$ and thus $P(x, \partial) \varphi=0$ for every $\varphi \in \mathcal{D}(\Omega), \varphi \neq 0$, with $\operatorname{supp}(\varphi) \cap \operatorname{supp}(\psi)=\varnothing$.

(2) The operator $P^{*}(x, \partial): \mathcal{D}^{*}\left(\mathbb{R}^{2}\right) \mapsto \mathcal{D}^{*}\left(\mathbb{R}^{2}\right), P^{*}(x, \partial)=x_{1} \frac{\partial}{\partial x_{2}}-x_{2} \frac{\partial}{\partial x_{1}}$ is nonregular, because $P(x, \partial)=-P^{*}(x, \partial)$ and $P(x, \partial) \varphi\left(x_{1}^{2}+x_{2}^{2}\right)=0$ for every $\varphi \in \mathcal{D}(\mathbb{R}), \varphi \neq 0$.

(3) For an example of a non-injective fourth order elliptic operator $P(x, \partial): \mathcal{D}\left(\mathbb{R}^{3}\right) \mapsto$ $\mathcal{D}\left(\mathbb{R}^{3}\right)$ with smooth (but non-analytic) coefficients, we refer to (Hörmander [14], Theorem 13.6.15). Its dual $P^{*}(x, \partial): \mathcal{D}^{*}\left(\mathbb{R}^{3}\right) \mapsto \mathcal{D}^{*}\left(\mathbb{R}^{3}\right)$ gives another example for a non-regular operator.

\section{Standardization of a nonstandard result}

Using Hamel bases, we show that the space of generalized distributions $\widehat{\mathcal{E}}(\Omega)$ introduced in (Todorov [37, § 2]) can be embedded as a $\mathbb{C}$-vector subspace into $\mathcal{D}^{*}(\Omega)$ (Example 7.6). Because $\widehat{\mathcal{E}}(\Omega)$ was defined in the framework of nonstandard analysis (Robinson [33]), we look upon $\mathcal{D}^{*}(\Omega)$ as a standardization of $\widehat{\mathcal{E}}(\Omega)$. Actually, our article itself can be viewed as a standardization of the article [37], because the surjectivity of the regular operators was first proved in [37] in the framework of $\mathcal{L}(\widehat{\mathcal{E}}(\Omega))$, while the main result of this article (Theorem 8.4) holds within $\mathcal{L}\left(\mathcal{D}^{*}(\Omega)\right.$ ). Thus, by replacing $\widehat{\mathcal{E}}(\Omega)$ by $\mathcal{D}^{*}(\Omega)$, 
our result about the regular operators becomes accessible even for readers without background in nonstandard analysis.

Example 12.1 (The Space $\widehat{\mathcal{E}}(\Omega)$ ) Let * $\mathcal{E}(\Omega)$ be the nonstandard extension of $\mathcal{E}(\Omega)=$ $\mathcal{C}^{\infty}(\Omega)$ in a $\mathfrak{c}_{+}$-saturated ultra-power nonstandard model with set of individuals $\mathbb{R}$ (Example 7.13 applied for $\kappa=\mathfrak{c}=$ card $\mathbb{R}$ ). Let $\widehat{\mathcal{E}}(\Omega)={ }^{*} \mathcal{E}(\Omega) / J_{\mathcal{D}(\Omega)}$ be the space of generalized distributions, where

$$
J_{\mathcal{D}(\Omega)}=\left\{f \in{ }^{*} \mathcal{E}(\Omega):(\forall \varphi \in \mathcal{D}(\Omega))\left(\int_{\Omega} f(x)^{*} \varphi(x) d x=0\right)\right\}
$$

and ${ }^{*} \varphi$ is the nonstandard extension of $\varphi$.

Recall that ${ }^{*} \mathcal{E}(\Omega)$ is a differential ring and ${ }^{\sigma} \mathcal{E}(\Omega)=\left\{{ }^{*} \varphi: \varphi \in \mathcal{E}(\Omega)\right\}$ is a differential subring of $* \mathcal{E}(\Omega)$. For our purpose we shall treat $* \mathcal{E}(\Omega)$ as a differential module over $\mathcal{E}(\Omega)$ under the product $\varphi f={ }^{*} \varphi \cdot f$, where $\varphi \in \mathcal{E}(\Omega)$ and $f \in{ }^{*} \mathcal{E}(\Omega)$. Here the dot in ${ }^{*} \varphi \cdot f$ stands for the point-wise product in ${ }^{*} \mathcal{E}(\Omega)$. Consequently, ${ }^{*} \mathcal{E}(\Omega)$ is a differential vector space over $\mathbb{C}$ as well, since $\mathbb{C} \subset \mathcal{E}(\Omega)$. We have (trivially) card $\widehat{\mathcal{E}}(\Omega) \leq \mathfrak{c}_{+}$, since $\operatorname{dim}\left({ }^{*} \mathcal{E}(\Omega)\right)=\operatorname{card}\left({ }^{*} \mathcal{E}(\Omega)\right)=\mathfrak{c}_{+}($Example 7.13 applied for $\kappa=\mathfrak{c}=\operatorname{card} \mathbb{R})$.

We observe that $J_{\mathcal{D}(\Omega)}$ is a differential $\mathcal{E}(\Omega)$-submodule of $* \mathcal{E}(\Omega)$ and also a differential $\mathbb{C}$-vector subspace of $* \mathcal{E}(\Omega)$ with the property ${ }^{\sigma} \mathcal{E}(\Omega) \cap J_{\mathcal{D}(\Omega)}=\{0\}$. Thus the mapping $f \mapsto{ }^{*} f+J_{\mathcal{D}(\Omega)}$ is a $\mathcal{E}(\Omega)$-module embedding as well as a $\mathbb{C}$-vector space embedding of $\mathcal{E}(\Omega)$ into $\widehat{\mathcal{E}}(\Omega)$. Among other things, this embedding implies card $\widehat{\mathcal{E}}(\Omega) \geq \mathfrak{c}$ and thus $\operatorname{dim} \widehat{\mathcal{E}}(\Omega)=\operatorname{card} \widehat{\mathcal{E}}(\Omega)$ due to the formula $\operatorname{card} \widehat{\mathcal{E}}(\Omega)=\max \{\operatorname{dim} \widehat{\mathcal{E}}(\Omega), \mathfrak{c}\}$ (Lemma 3.3). The inequality $c \leq \operatorname{dim} \widehat{\mathcal{E}}(\Omega) \leq \mathfrak{c}_{+}$implies that either $\operatorname{dim} \widehat{\mathcal{E}}(\Omega)=\mathfrak{c}$, or $\operatorname{dim} \widehat{\mathcal{E}}(\Omega)=\mathfrak{c}_{+}$. In either case $\widehat{\mathcal{E}}(\Omega)$ can be embedded as a $\mathbb{C}$-vector subspace of $\mathcal{D}^{*}(\Omega)$ by Lemma 3.9, since $\operatorname{dim} \mathcal{D}^{*}(\Omega)=\mathfrak{c}_{+}$(Example 7.6).

Standardizations of nonstandard results are fascinating and often dramatic events in mathematics:

- The most famous example of standardization is certainly the creation of the modern Calculus, which standardizes the old Leibniz-Newton-Euler Infinitesimal Calculus. Getting rid of infinitesimals and replacing them with limits can be described as nothing less than a real drama, or even revolution in mathematics (Hall and Todorov [10]). The drama took several decades and it resulted-as side products - in the rigorous theory of real numbers used today, set theory and mathematical logic. For those interested in the history of infinitesimals in the context of the Reformation, Counter-Reformation and English Civil War, we refer to the excellent book by Alexander [1]. 
- A more recent example of a standardization of a nonstandard result comes from the Bernstein-Robinson [3] solution of the open Invariant Subspace Problem for polynomially-compact operators on Hilbert space. Here the drama was not missing either. Paul Halmos [11]—in the role of Editor/Referee-decided to delay the acceptance of Bernstein-Robinson's manuscript for almost a year, and meanwhile to translate (after sweating on it, by his own words) the submitted manuscript into the language of standard analysis, in an almost line-by-line correspondence to the original. Eventually, Halmos published his translation side by side with Bernstein-Robinson's article in the same issue of the Pacific Journal of Mathematics. Halmos's pseudo-standardization of the Bernstein-Robinson solution (of an open until then problem in mathematics) considerably deflected the expected impact of one of the first and perhaps the most spectacular application of the newly-born Nonstandard Analysis. Bitter feelings about this episode are still lingering in the nonstandard community. The real standardization of Bernstein-Robinson's solution came almost a decade later from Lomonosov [26] in the framework of a more advanced and general operator theory.

\section{Acknowledgements}

The author thanks Michael Oberguggenberger and Ivan Penkov for reading the earlier versions of the manuscript and for the insightful dialog. The author is also grateful to the anonymous referee for the numerous useful suggestions.

\section{References}

[1] A Alexander, Infinitesimals: How a Dangerous Mathematical Theory shaped the Modern Would, Scientific American/Farrar, Straus and Giroux, New York (2014)

[2] S Axler, Linear Algebra Done Right, third edition, Undergraduate Texts in Mathematics, Springer, Cham (2015); https://doi.org/10.1007/978-3-319-11080-6

[3] A Bernstein, A Robinson, Solution of an invariant subspace problem of K. T. Smith and P. R. Halmos., Pacific Journal of Mathematics 16 (1966) 421-431; https://doi.org/ 1102994835

[4] C C Chang, H J Keisler, Model Theory, third edition, Dover Books on Mathematics, Dover Publications, New York (2012)

[5] A Chirvasitu, I Penkov, Representation categories of Mackey Lie algebras as universal monoidal categories, Pure and Applied Mathematics Quarterly 13 (2017) 77-121; https://doi.org/10.4310/PAMQ.2017.v13.n1.a3 
[6] J F Colombeau, Elementary Introduction to New Generalized Functions, volume 113 of North-Holland Mathematical Studies, North-Holland Publishing Company, Amsterdam (1985)

[7] J F Colombeau, A Heibig, M Oberguggenberger, Le problemé de Cauchy dans un espace de fonctions généralisées I, Comptes Rendus de l'Académie des Sciences Series I — Mathematics 317 (1993) 851-855

[8] M Davis, Applied Nonstandard Analysis, Dover Books on Mathematics, Dover Publications, New York (2005)

[9] L Ehrenpreis, Solutions of some problems of division III. Division in the spaces, $\mathcal{D}^{\prime}, \mathcal{H}$, $\mathcal{Q}_{A}, \mathcal{O}$, American Journal of Mathematics 78 (1956) 685-715; https://doi.org/10.2307/ 2372464

[10] J F Hall, T D Todorov, Ordered Fields, the Purge of Infinitesimals from Mathematics and the Rigorousness of Infinitesimal Calculus, Bulgarian Journal of Physics 42 (2015) 99-127. An issue dedicated to the centenary of the birth of Prof. Christo Yankov Christov. arXiv:1509.03798

[11] P R Halmos, Invariant subspaces of polynomially compact operators, Pacific Journal of Mathematics 16 (1966) 433-437

[12] G Hamel, Eine Basis aller Zahlen und die unstetige Lösungen der Funktional gleichung $f(x+y)=f(x)+f(y)$, Mathematische Annalen 60 (1905) 459-462; https://doi.org/ $10.1007 / \mathrm{BF} 01457624$

[13] E Hewitt, K Stromberg, Real and abstract analysis. A modern treatment of the theory of functions of a real variable, Springer-Verlag, New York (1965)

[14] L Hörmander, The Analysis of Linear Partial Differential Operators II: Differential Operators with Constant Coefficients, Classics in Mathematics, Springer-Verlag, Berlin (1983). Originally published as Vol. 257 in the series: Die Grundlehren der mathematischen Wissenschaften. https://doi.org/10.1007/b138375

[15] L Hörmander, The Analysis of Linear Partial Differential Operators I: Distribution Theory and Fourier Analysis, Classics in Mathematics, Springer-Verlag, Berlin (1990). Originally published as Vol. 256 in the series: Die Grundlehren der mathematischen Wissenschaften. https://doi.org/10.1007/978-3-642-61497-2

[16] L Hörmander, The Analysis of Linear Partial Differential Operators III: PseudoDifferential Operators, Classics in Mathematics, Springer-Verlag, Berlin (1994). Originally published as Vol. 274 in the series: Die Grundlehren der mathematischen Wissenschaften. https://doi.org/10.1007/978-3-540-49938-1

[17] L Hörmander, The Analysis of Linear Partial Differential Operators IV: Fourier Integral Operators, Classics in Mathematics, Springer-Verlag, Berlin (1994). Originally published as Vol. 276 in the series: Die Grundlehren der mathematischen Wissenschaften. https://doi.org/10.1007/978-3-642-00136-9 
[18] T W Hungerford, Algebra, volume 73 of Graduate Texts in Mathematics, SpringerVerlag, New York-Berlin (1980). Reprint of the 1974 original. https://doi.org/10.1007/ 978-1-4612-6101-8

[19] N Jacobson, Lectures in Abstract Algebra II. Linear Algebra, volume 31 of Graduate Texts in Mathematics, Springer-Verlag, New York-Berlin (1975). Reprint of the 1953 edition. https://doi.org/10.1007/978-1-4684-7053-6

[20] T J Jech, The Axiom of Choice, Dover Books on Mathematics, Dover Publications, New York (2008)

[21] A Kaneko, Introduction to Hyperfunctions, volume 3 of Mathematics and its Applications (Japanese Series), Kluwer Academic Publishers Group, Tokyo (1988). Translated from the Japanese by Y. Yamamoto.

[22] G Köthe, Topologische Lineare Räume, Vol. I, volume 107 of Die Grundlehren der mathematischen Wissenschaften, second edition, Springer-Verlag, Berlin-New York (1966); https://doi.org/10.1007/978-3-662-22555-4

[23] H Lewy, An example of a smooth linear partial differential equation without solution, Annals of Mathematics 66 (1957) 155-158; https://doi.org/10.2307/1970121

[24] M J Lighthill, Introduction to Fourier analysis and generalised functions, Cambridge University Press, New York (1960); https://doi.org/10.1017/CBO9781139171427

[25] T Lindstrøm, An invitation to nonstandard analysis, from: "Nonstandard Analysis and its Applications", London Mathematical Society Student Texts 10, Cambridge University Press, Cambridge (1988) 1-105; https://doi.org/10.1017/CBO9781139172110.002

[26] V I Lomonosov, Invariant subspaces of the family of operators that commute with a completely continuous operator, Functional Analysis and Its Applications 7 (1973) 55-56; https://doi.org/10.1007/BF01080698

[27] G W Mackey, On infinite-dimensional linear spaces, Transactions of the American Mathematical Society 57 (1945) 155-207; https://doi.org/10.1090/S0002-9947-19450012204-1

[28] B Malgrange, Existence et approximation des solutions des équations aux dérivées partielles et des équations de convolution, Annales de l'Institut Fourier 6 (1955-56) 269-354; https://doi.org/10.5802/aif.65

[29] M Oberguggenberger, Multiplication of Distributions and Applications to Partial Differential Equations, volume 259 of Pitman Research Notes in Mathematics Series, Longman Scientific \& Technical, Harlow (1992)

[30] M Oberguggenberger, On the algebraic dual of $\mathcal{D}(\Omega)(2013)$; arXiv: 1304.2512

[31] M Oberguggenberger, E Rosinger, Solution of Continuous Nonlinear PDEs through Order Completion, volume 181 of North-Holland Mathematics Studies, North-Holland Publishing Company, Amsterdam (1994)

[32] M Oberguggenberger, T D Todorov, Solving linear partial differential equations in $\mathcal{D}^{*}$ (1996); unpublished manuscript. 
[33] A Robinson, Non-standard Analysis, North-Holland Publishing Company, Amsterdam (1966)

[34] E E Rosinger, Non-linear Partial Differential Equations. An Algebraic View of Generalized Solutions, volume 164 of North-Holland Mathematics Studies, North-Holland Publishing Company, Amsterdam (1990)

[35] P Schapira, Une équation aux dérivées partielles sans solutions dans l'espace des hyperfonctions, Comptes Rendus de l'Académie des Sciences - Series I — Mathematics 265 (1967) 665-667; https://doi.org/10.24033/bsmf.1679

[36] B Street, What else about ... hypoellipticity?, Notices of the American Mathematical Society 65 (2018) 424-425

[37] T Todorov, An Existence Result for a Class of Partial Differential Equations with Smooth Coefficients, from: "Advances in Analysis, Probability and Mathematical Physics; Contributions to Nonstandard Analysis", (S Albeverio, W A J Luxemburg, M P H Wolff, editors), Mathematics and Its Applications 314, Kluwer Academic Publishers Group, Dordrecht (1995) 107-121; https://doi.org/10.1007/978-94-015-8451-7_9

[38] F Tréves, On Local Solvability of Linear Partial Differential Equations, Bulletin of the American Mathematical Society 76 (1970) 552-571; https://doi.org/10.1090/S00029904-1970-12443-0

[39] V S Vladimirov, Generalized Functions in Mathematical Physics, Mir, Moscow (1979)

[40] R S Wolf, Proof, Logic, and Conjecture: The Mathematician's Toolbox, W.H. Freeman and Company, New York (1998)

Professor Emeritus, California Polytechnic State University, San Luis Obispo, California 93407, USA.

ttodorov@calpoly.edu

https://math.calpoly.edu/todor-todorov

Received: 11 May 2019 Revised: 18 March 2021 